\title{
Cloud computing security taxonomy: From an atomistic to a holistic view
}

\section{Siyakha N. Mthunzi}

\section{Elhadj Benkhelifa ${ }^{a, b, *}$}

e.benkhelifa@staffs.ac.uk

\section{Tomasz Bosakowsk}

Chirine Ghedira

Mahmoud Barhamgi

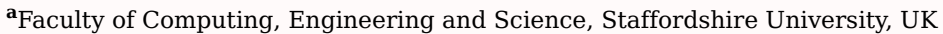

${ }^{\mathbf{b}}$ Cloud Computing Research Group, College Road, Stoke-on-Trent, Staffordshire, ST4 2DE, UK

'Staffordshire University, Stoke on Trent, UK

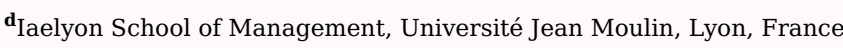

eUniversité Claude Bernard, Lyon 1, France

${ }^{*}$ Corresponding author at: Faculty of Computing, Engineering and Science, Staffordshire University, UK.

\section{Abstract}

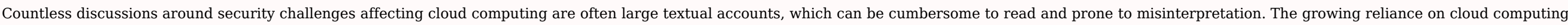

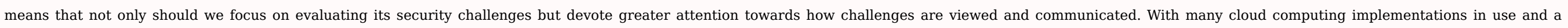

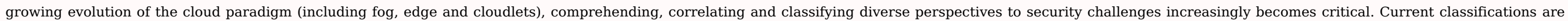

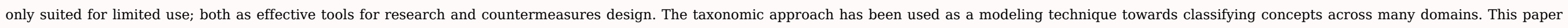

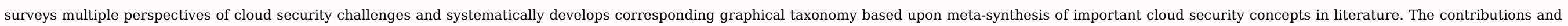

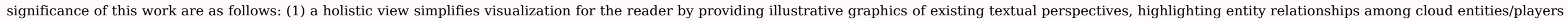

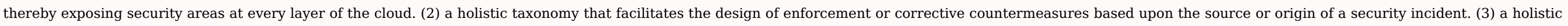
taxonomy highlights security boundary and identifies apt areas to implement security countermeasures.

\section{Introduction}

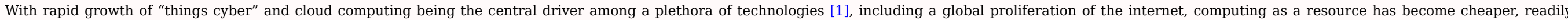

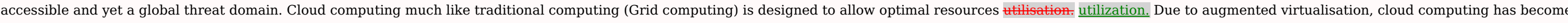

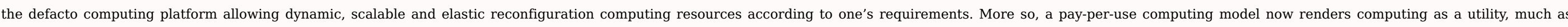

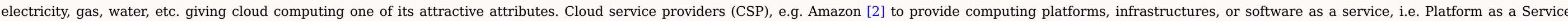

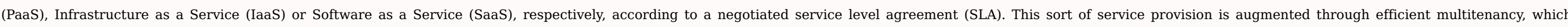

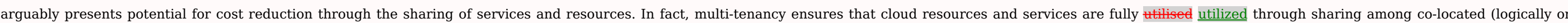




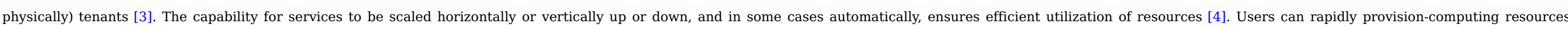

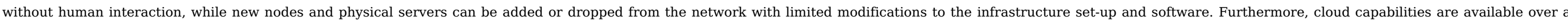

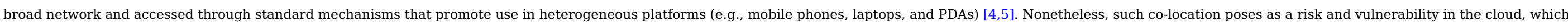
is further complicated by interactions (which are often dynamic, unpredictable, transient and intensive) among different service domains driven by service requirements.

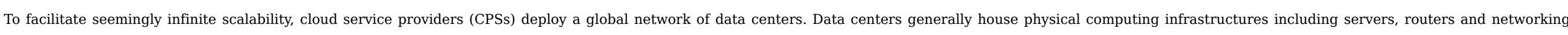

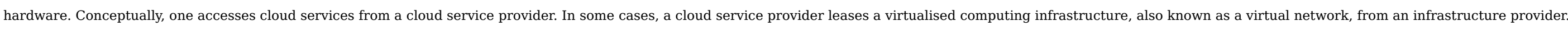

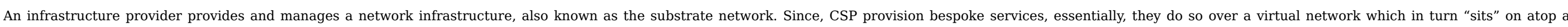

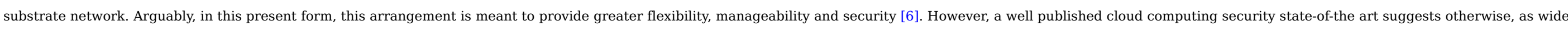

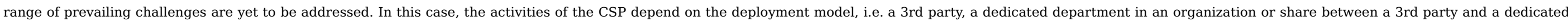
department such as in community or hybrid clouds.

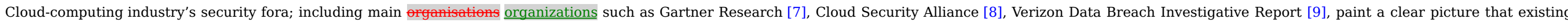

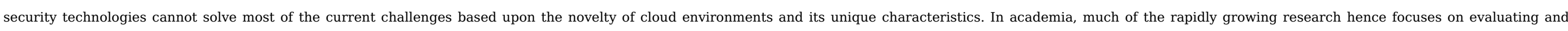

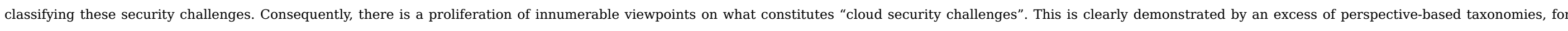

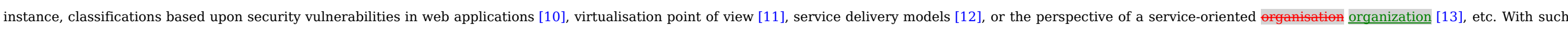

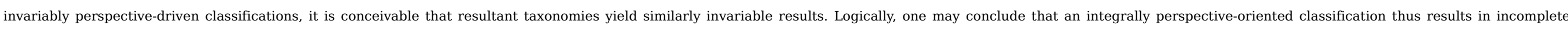

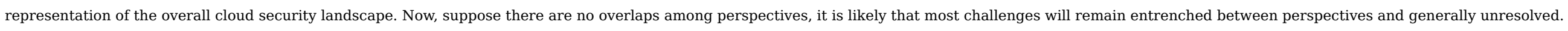

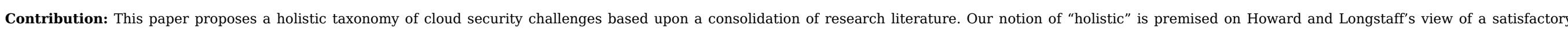

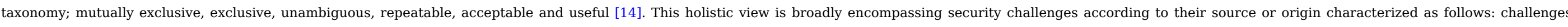

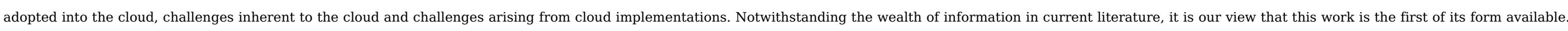

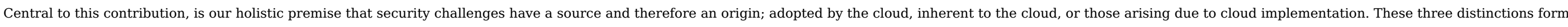
the root of the proposed taxonomy.

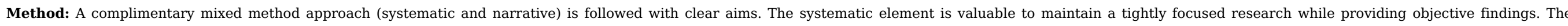

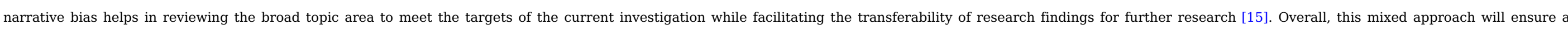

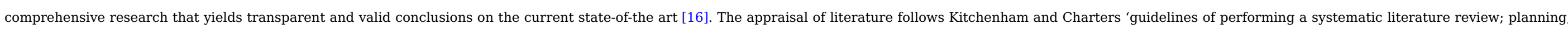

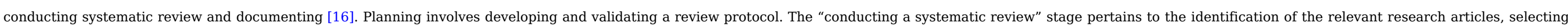
primary studies, assessing the quality of studies and extracting study data. Furthermore, "documentation" relates to the construction of the reviews, including the discussions that arise.

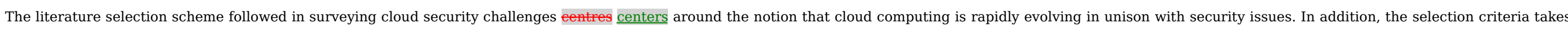

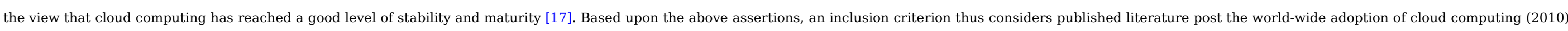

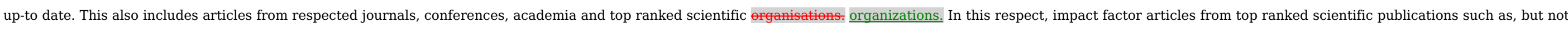

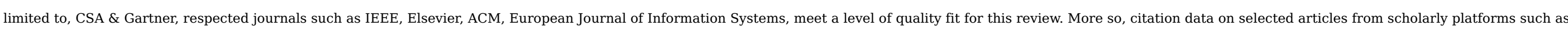

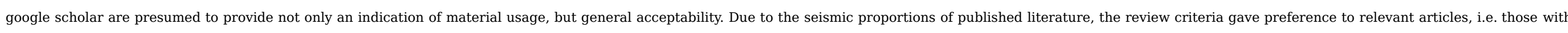

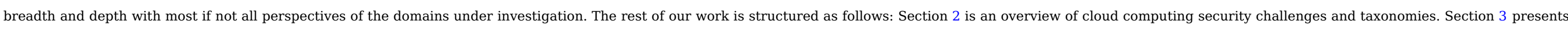

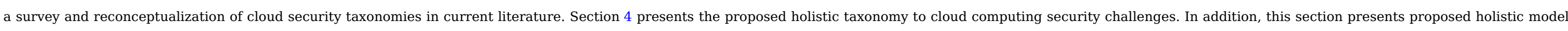

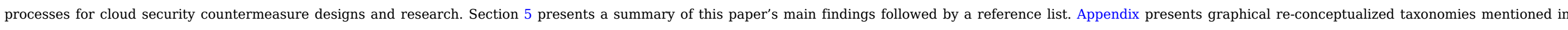
Section 3 above.

\section{Cloud computing security}




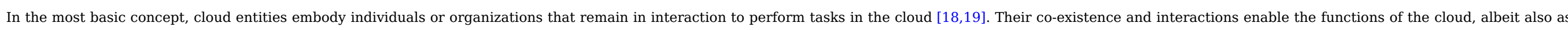

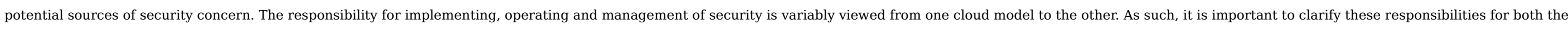

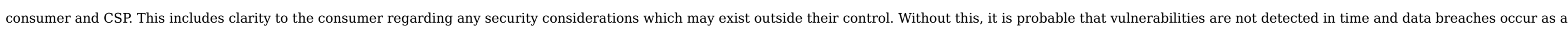
result.

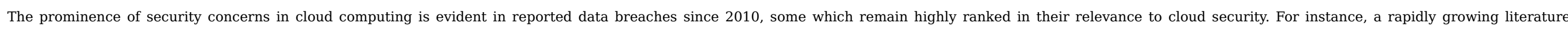

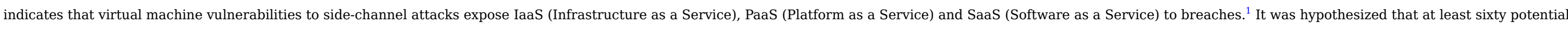

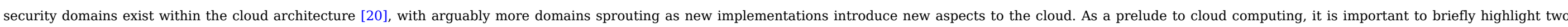

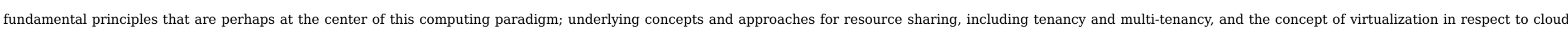

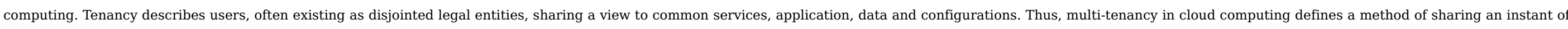

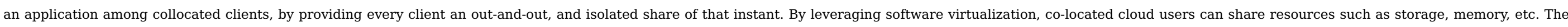

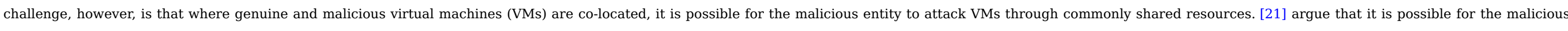

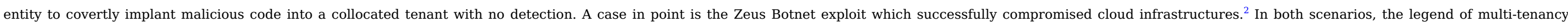

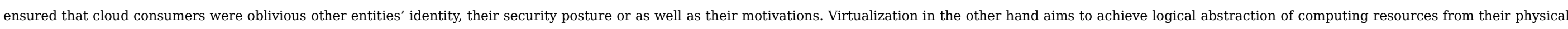

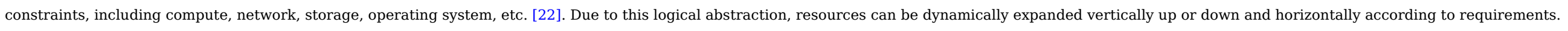

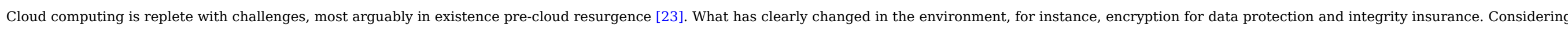

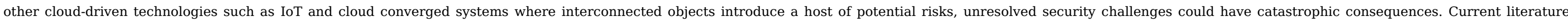

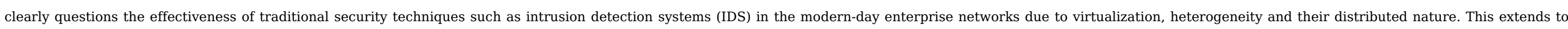

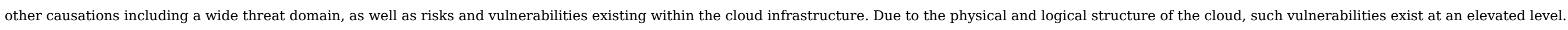

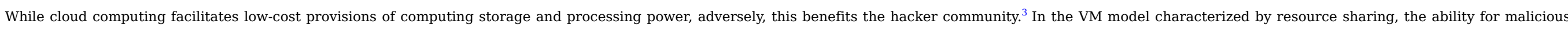

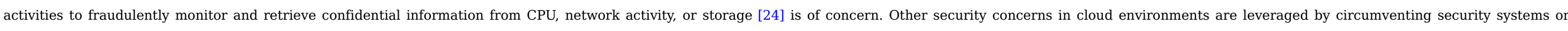

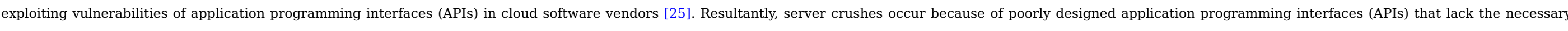

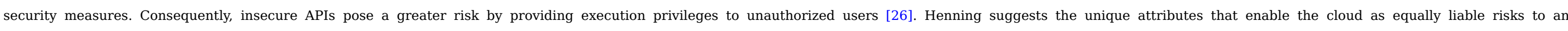
organization [27].

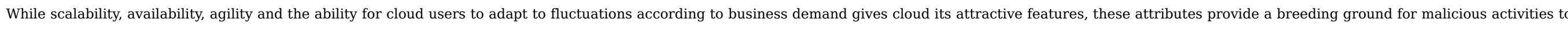

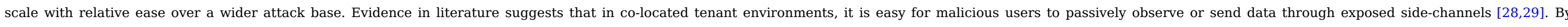

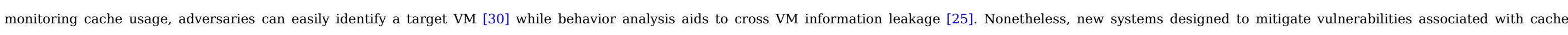
monitoring exist. In fact, [31] suggests datacenter virtualization and middleware sharing to be efficient approaches for a multi-tenant solution, i.e. one application instance for each tenant.

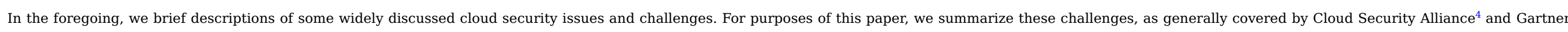

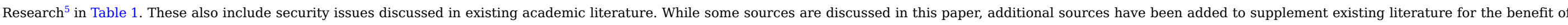
the interested reader.

Table 1 Summary of cloud security challenges.

\begin{tabular}{|c|c|c|}
\hline Challenge & Issue & Reference \\
\hline Control & $\begin{array}{l}\text { Outsourcing, PaaS, SaaS and IaaS. Ease of use by end user's degree of information security \& control, i.e. control of data \& control over security } \\
\text { - Data owner has no full physical control over their data } \\
\text { - No control over OSs, network \& servers, storage \& applications in SaaS }\end{array}$ & {$[32,33]$} \\
\hline
\end{tabular}


- No control over Oss, network \& servers in PaaS

- Loss of control over networking components in IaaS

Trust

Virtualization

Malware

Attacks on web services

Denial of service

Weak identity, credential \& access management

Data breaches

Data loss

Insecure interfaces and APIs

Account \& service traffic hijacking
Securely establishing trust between servers \& clients, \& trusting cloud environments.

- Trust between servers $\&$ the clients misuse of cloud services

- Impose security policies

Updating security countermeasures is paramount to preventing data breaches \& leaks

- Risk to integrity of saved VM images

- Malicious insider

- Risks to confidential data stored in virtual machines

Intrusive and hostile software

- Sophisticated malware such as Stuxnet \& Flame.

- Zero-day exploits

Prominent attacks \& immature coding exploit online vulnerabilities

- Injection flaws \& cross-site scripting

- Signature wrapping attacks

- Malware, CSS, and Denial of Service (DoS)

Compromise the availability of services

- Semantic \& flooding DDoS attacks

- FRC attacks falsely use cloud resources: impacts application-layer servers

- Exploits are financially detrimental to a cloud consumer

- Cause the Operating System (OS) kernel to crash

\section{nsufficient scalability in identity access management systems}

- Spoofing attacks

- DoS attacks

- Elevations of privileges

- Repudiation

Unauthorised Unauthorizedaccess/use of confidential/sensitive data.

- Vulnerabilities in applications

- Malicious insider.

- Information disclosure

Insecure \& unnoticed configurations or vulnerabilities result in potential exploits \& data loss

- Side-channel attacks expose IaaS, PaaS and SaaS to breaches

Poorly designed APIs in cloud software vendors

- Provide execution privileges to unauthorised unauthorized users

Exploitation of software weaknesses, and personal information

- Phishing attack

- Service or account hijacking
$[5,11,34]$.

[35-38]

[39-42]

[43-48]

$[32,41,42,49-51]$

$[21,53,54]$

$[49,55]$

$[49,56]$ 
- Co-residency attacks

- Malicious insider

Abuse of cloud resources

Where IaaS providers lose some control

- Hackers \& spammers take advantage of free limited trials

Choosing and moving functions to cloud environments entails careful consideration

$49,59,60]$

Insufficient due diligence

Shared technology vulnerabilities

Privileged user access

Data location

Regulatory compliance

Data segregation

Recovery

Long term viability
Vulnerabilities due to intrinsic \& prevalent core cloud computing technologies

Where 3rd parties process data outside the enterprise

- Outsourcing means that an enterprise's security controls (physical or logical) are bypassed.

Location of data \& processes where routine maintenance is provided by a cloud vendor,

- No information to the cloud consumer about where their data is held,

as well as what processes are being performed

Challenges when dealing with LSAs \& other process \& regulatory issues

- Assurance

- Process and regulatory issues

Logical storage of data in multi-tenant environments

- Cross channel attacks

- Malicious insider

Low-cost disaster recovery \& data storage solutions

Concerns a cloud consumer's data if a CSP loses their business

\section{Existing cloud security taxonomies}

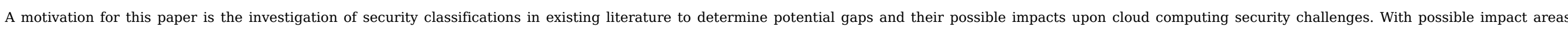

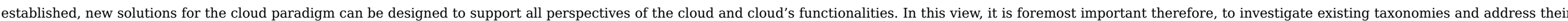

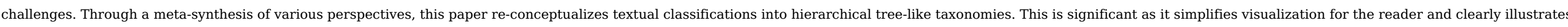

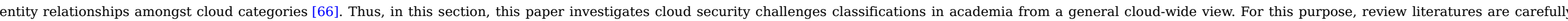

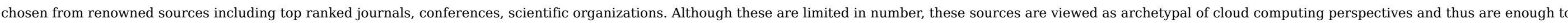
constitute a satisfactory security review. In this section, we distinguish:

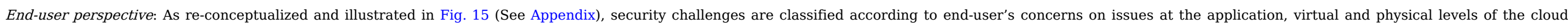

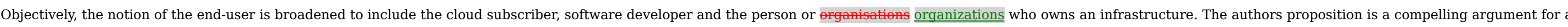

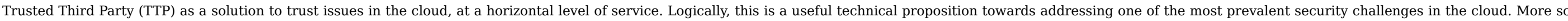

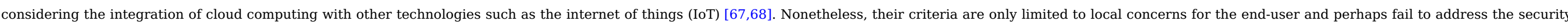

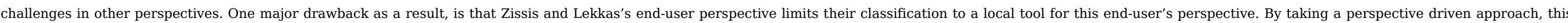

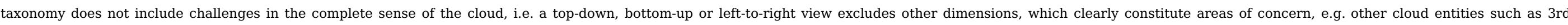
parties.

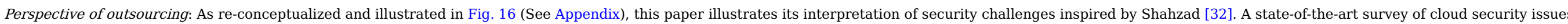




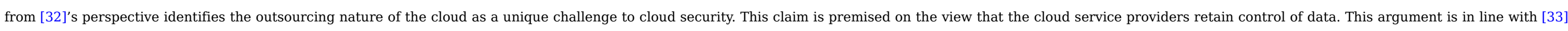

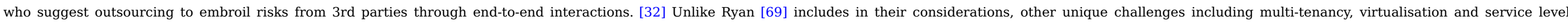

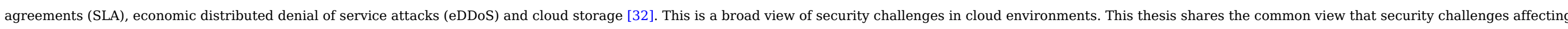

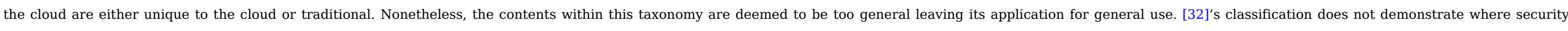
issues impact the cloud, which layer of the cloud, at what level, etc.

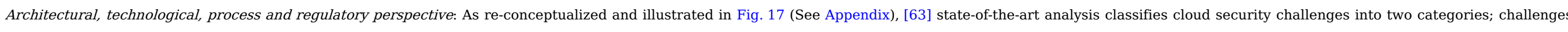

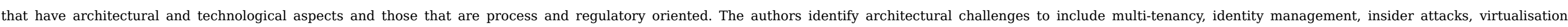

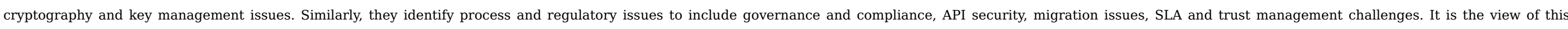

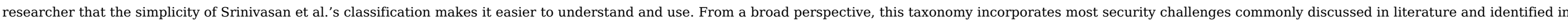

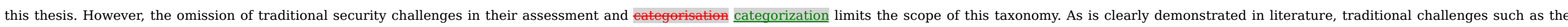
DoS, malware, malicious insider, etc. remain a threat to confidentiality, integrity and privacy in the cloud paradigm.

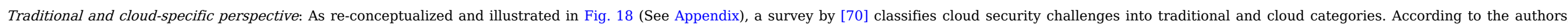

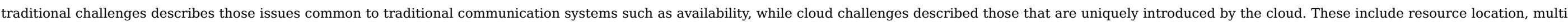

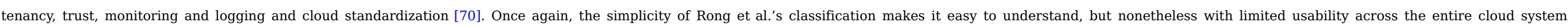

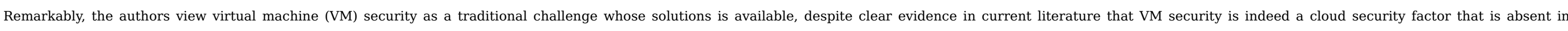

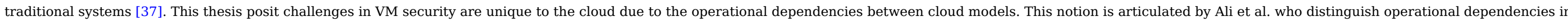

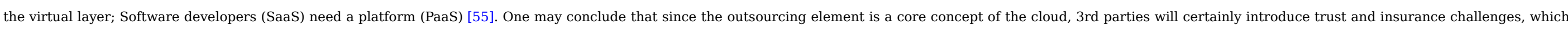
are unique to the cloud.

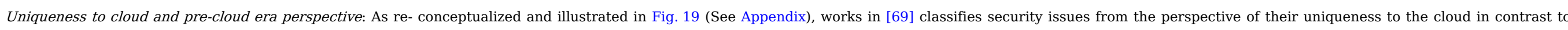

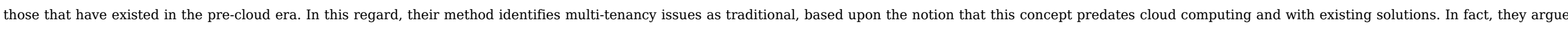

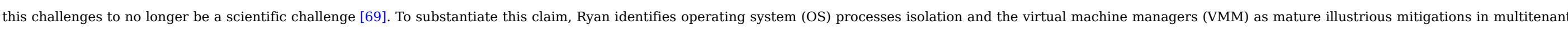

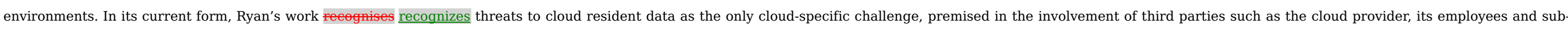

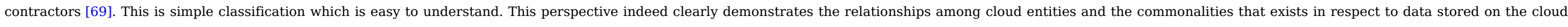

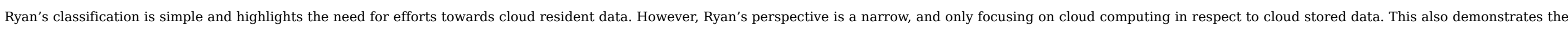
non-exhaustiveness of Ryan's taxonomy, as it misses key cloud components and issues around the virtual machine monitor (VMM), trust, control, legal, process and regulatory issues.

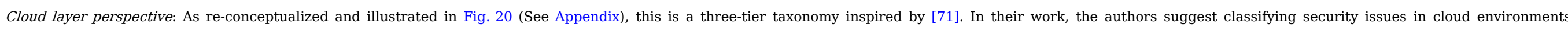

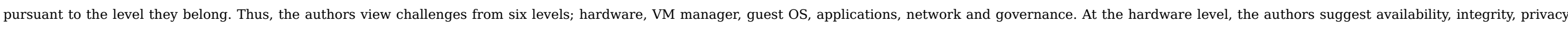

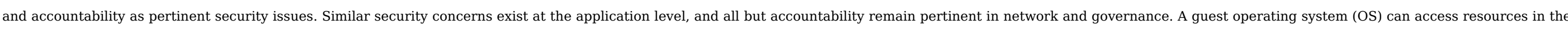

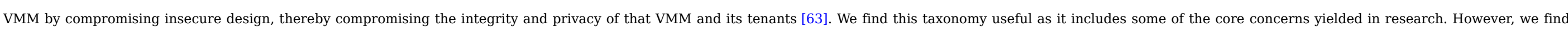

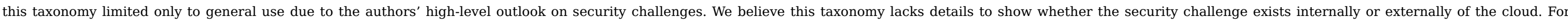

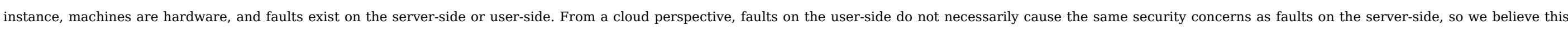
taxonomy would benefit from further detailing.

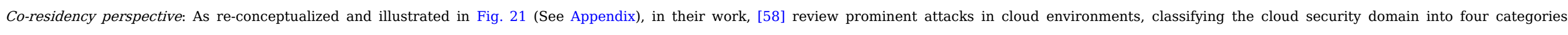

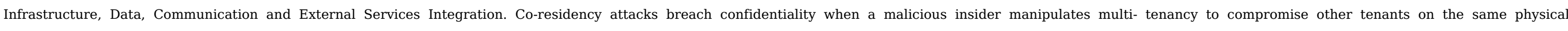

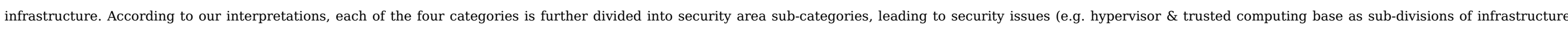

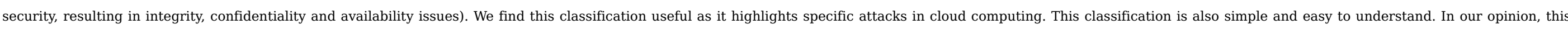




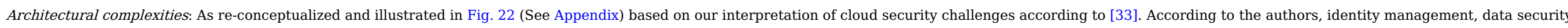

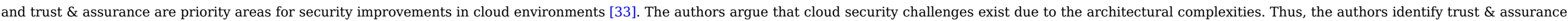

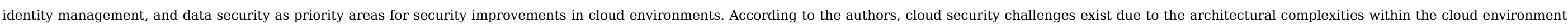

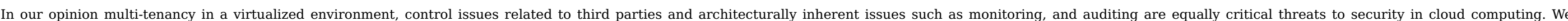
therefore find this taxonomy limited as it does not include some critical security challenges.

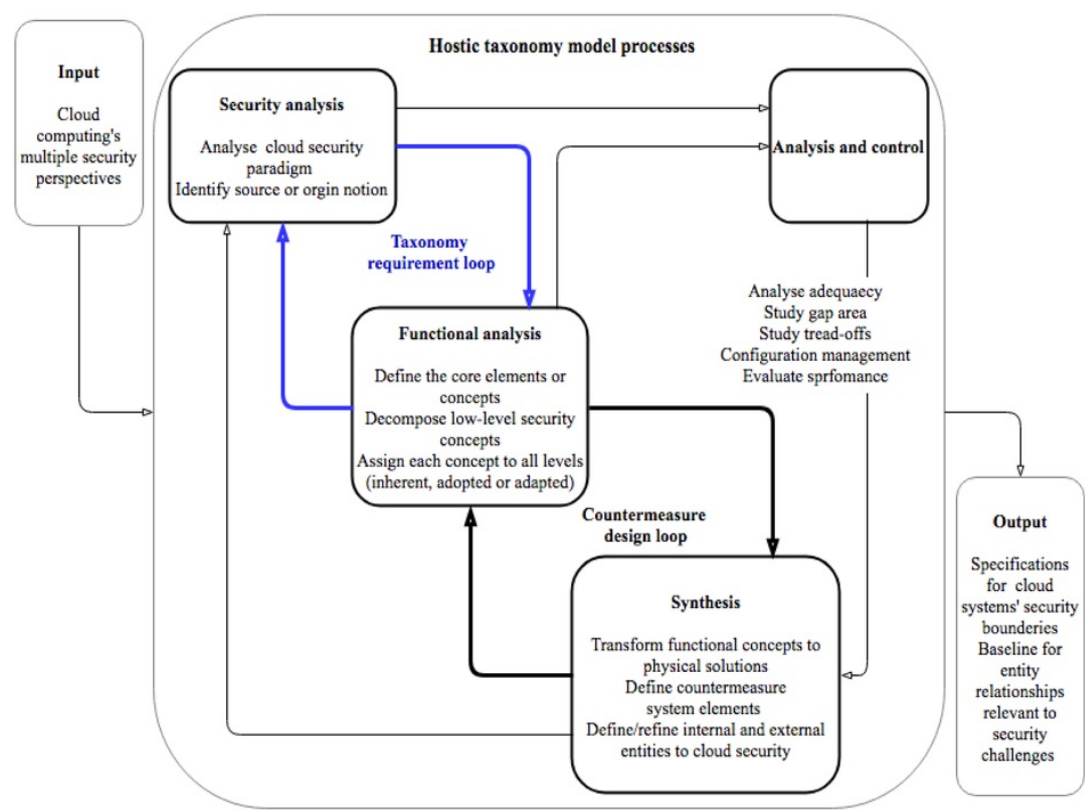

Fig. 1 An illustration of a generic holistic taxonomy development model processes.

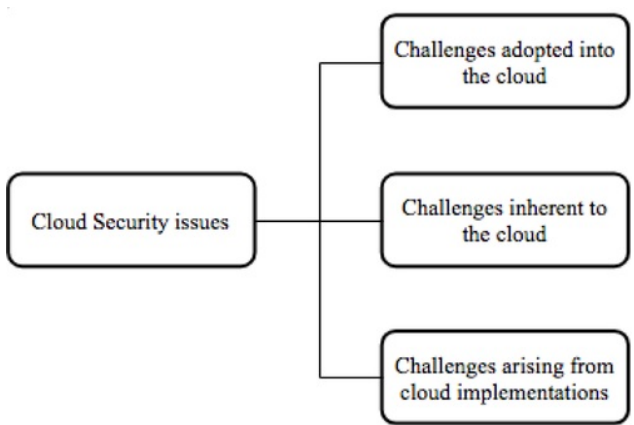

Fig. 2 High-level illustration of the holistic view to cloud computing security challenges.

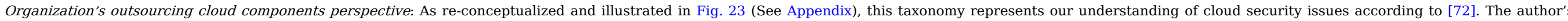

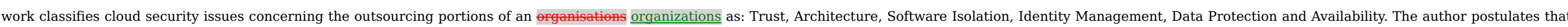




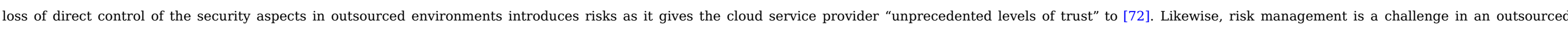

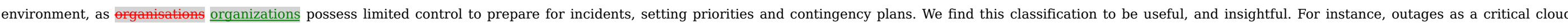

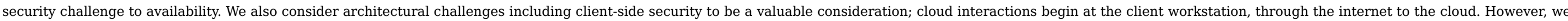
believe that this classification could benefit from illustrating wider coverage. For instance, considering trust issues pertinent to the consumer vs trust issues pertinent to a 3rd party such as a sub-contractor.

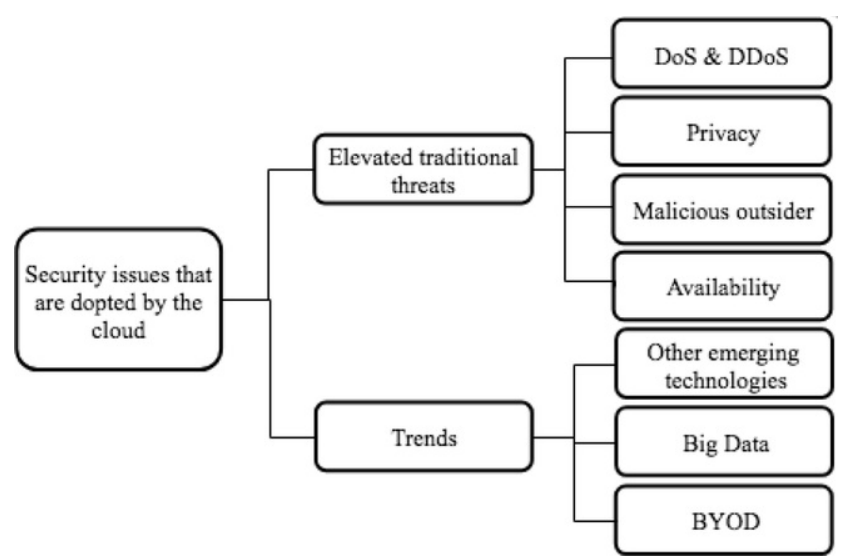

Fig. 3 Taxonomy on unique adopted security issues.

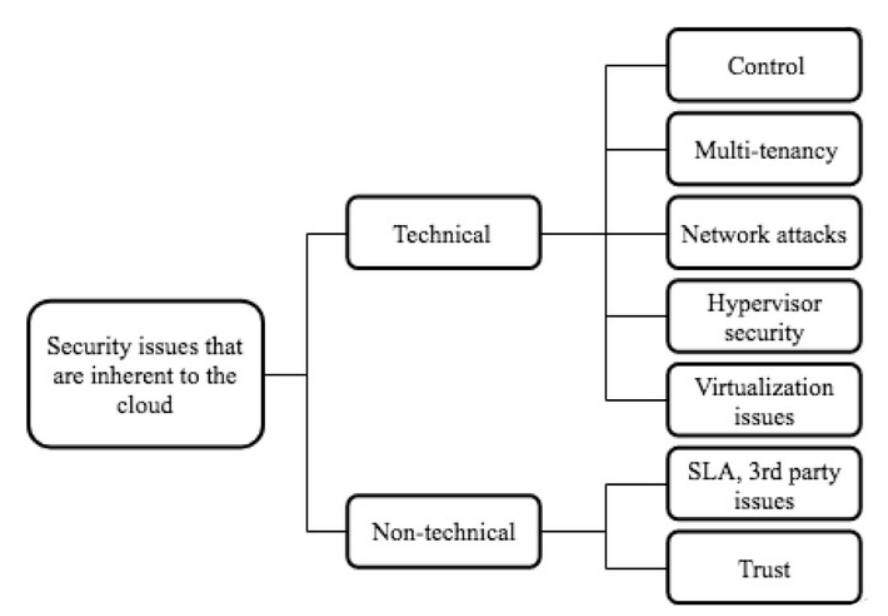

Fig. 4 Taxonomy of security issues inherent to the cloud. 


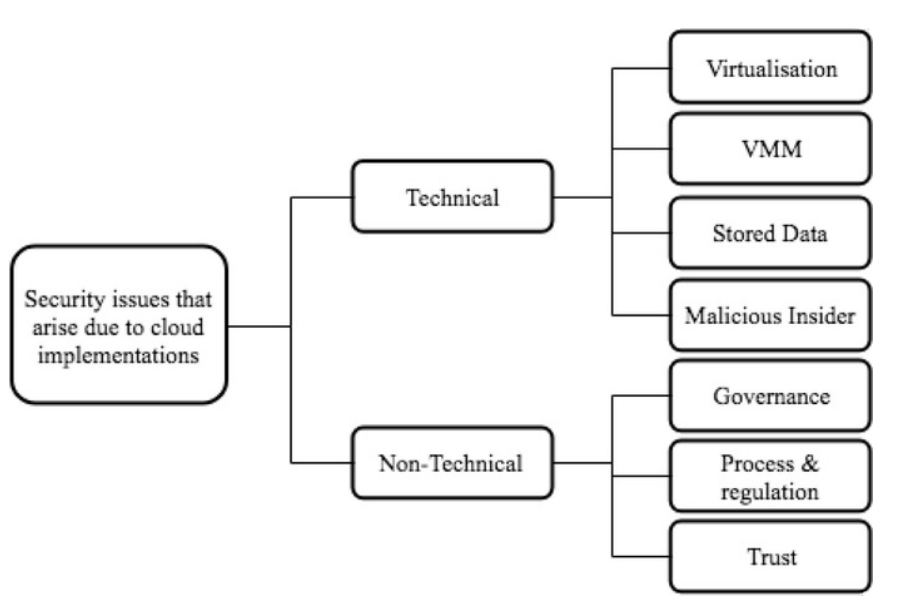

Fig. 5 Taxonomy of unique security issues arising from implementations in the cloud.

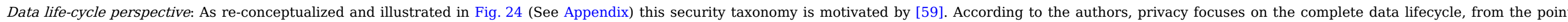

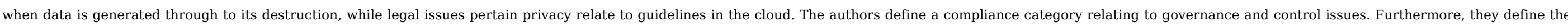

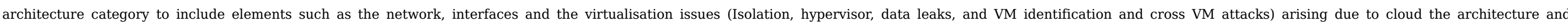
infrastructural implementations. According to the authors, other challenges include decision making, user access and authentication.

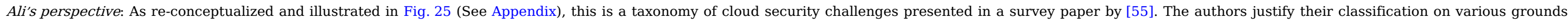

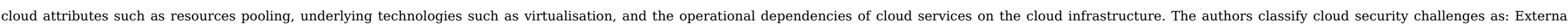

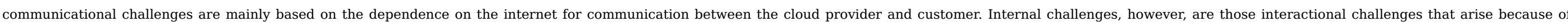

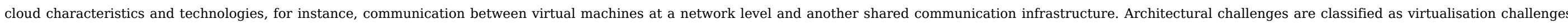

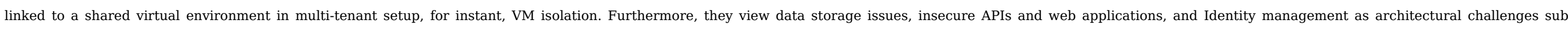

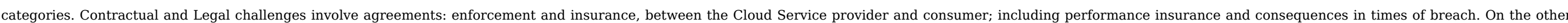
hand, Legal challenges include issues involving discrepancies in the application of the law due to physical locations and/or jurisdiction.

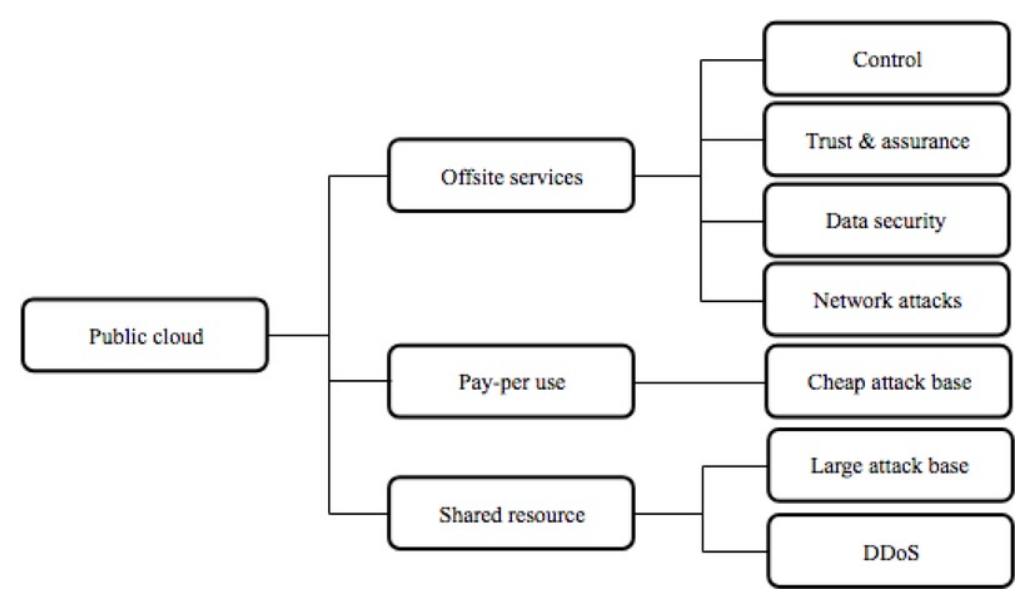

Fig. 6 Taxonomy of unique security issues in public clouds. 


\subsection{Analysis}

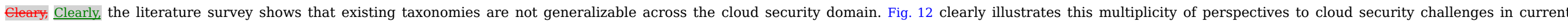

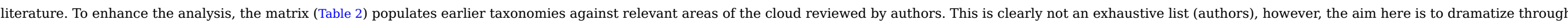

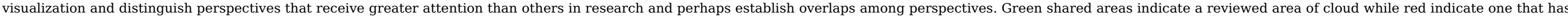
not been reviewed.

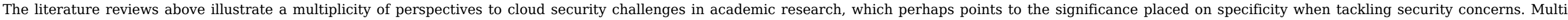

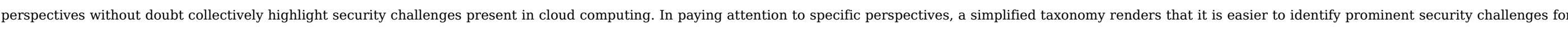

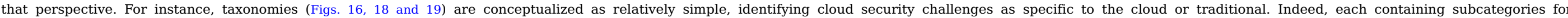

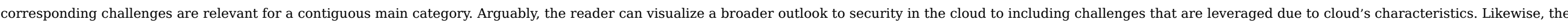

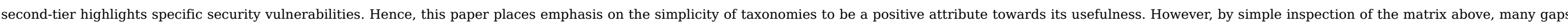

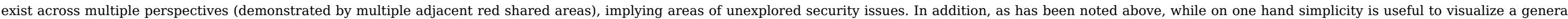
outlook, simplicity lacks specific and deeper detail, and hence an inherent deficiency which renders such taxonomies inadequate as tools for developing comprehensive solutions.

Table 2 A sample of specific topics areas and references covered in articles cloud security challenges (see [73]).

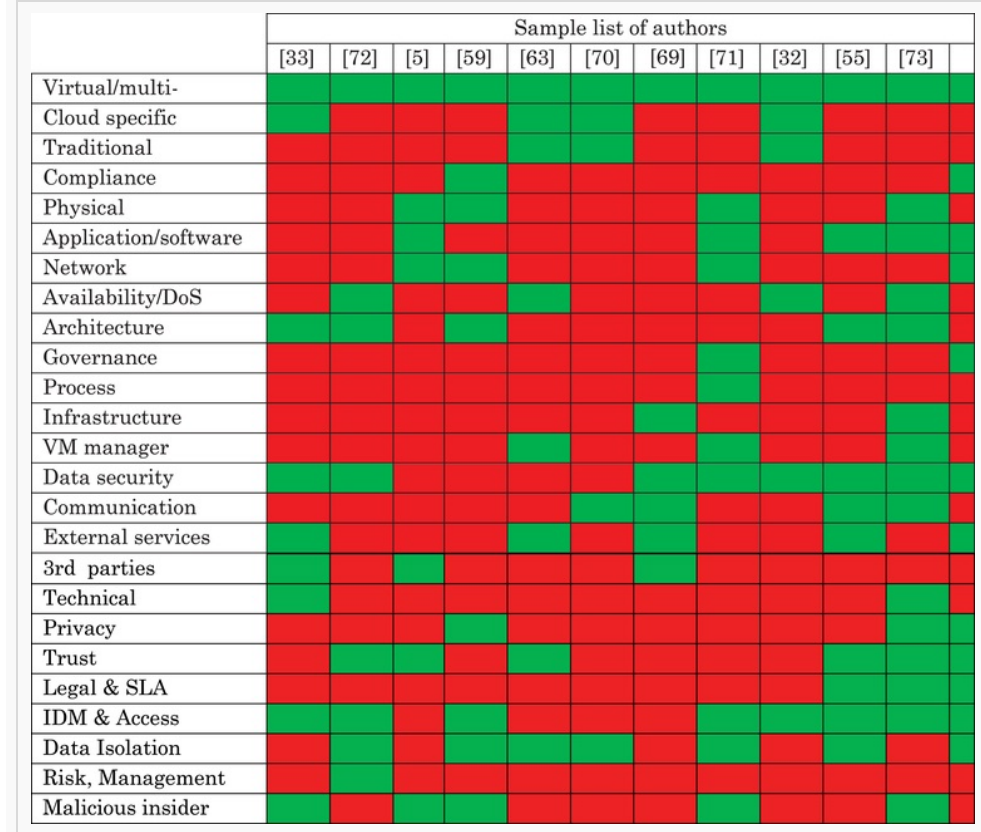

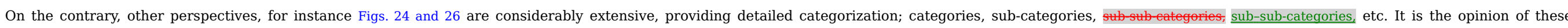

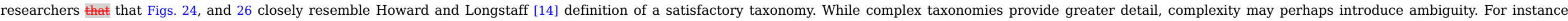

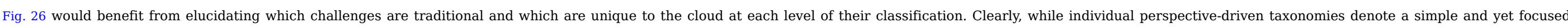

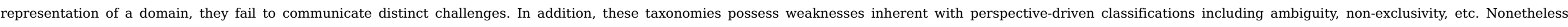

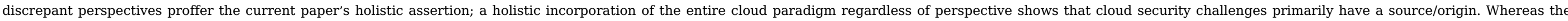

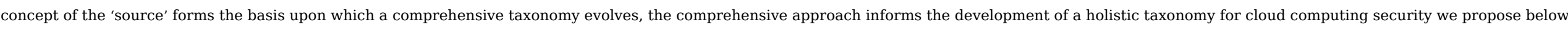




\section{A holistic taxonomy of cloud security challenges}

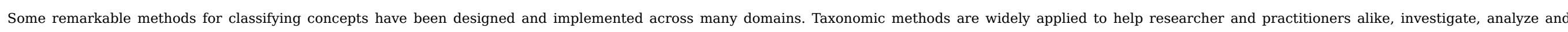

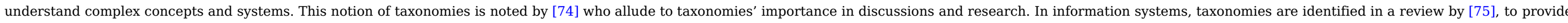

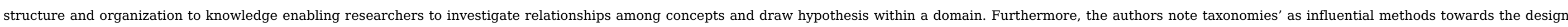

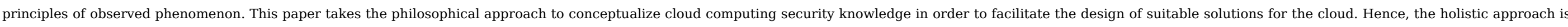
along that of Howard \& Longstaff encompassing mutual exclusivity, exhaustivity, unambiguity, repeatability, acceptability and usefulness of a satisfactory taxonomy [14], these are summarized as follows:

- Mutual exclusivity: a single subject cannot exist in multiple categories

- Exhaustivity: all possible classes are represented in the taxonomy

- Unambiguity: the presentation of categories should be consistent and clear in order to provide a complete classification

- Repeatability: An element which features more than once should always feature in the same category in all circumstances

- Acceptability: To be generally acceptable, the categories should be consistent and intuitive.

- Usefulness: Should provide greater insight into the subject topic.

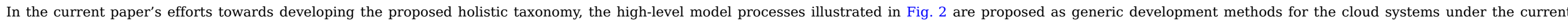
consideration. For the interested reader, a range of commonly applied taxonomy development methods exist in literature; [75] presents an interesting cross-domain review of us (see Fig. 1).

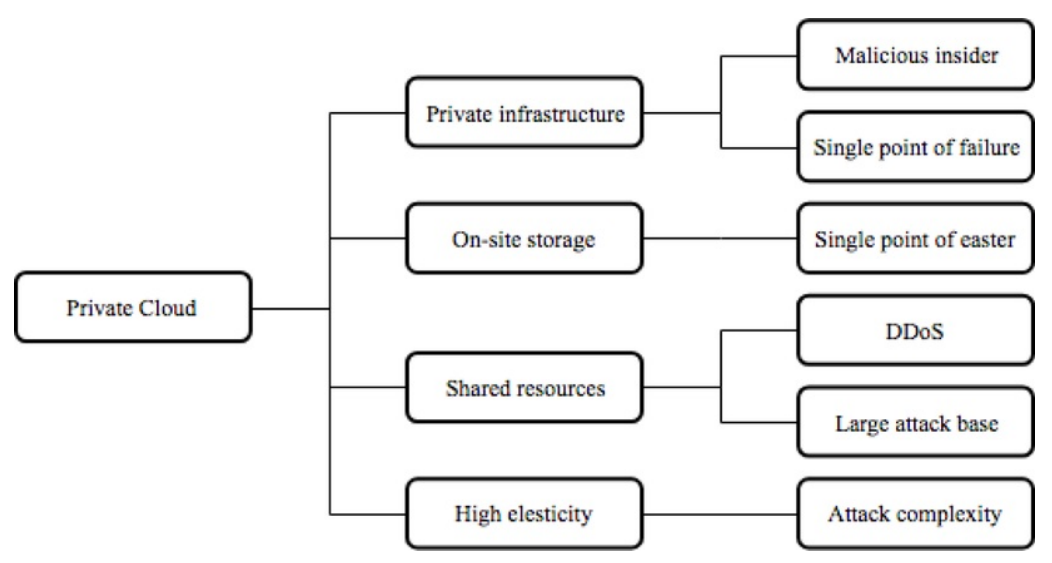

Fig. 7 Taxonomy of unique security issues in private cloud. 


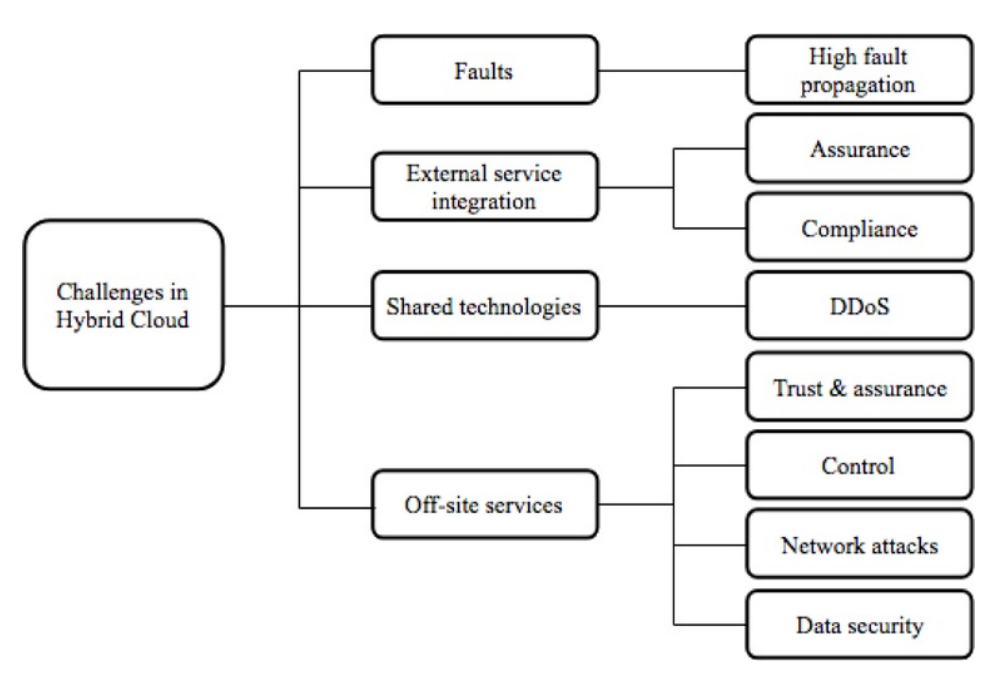

Fig. 8 Taxonomy of unique security issues in hybrid cloud.

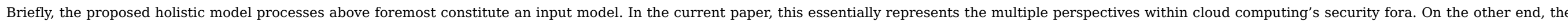

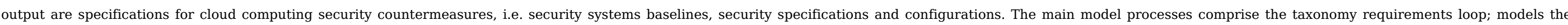

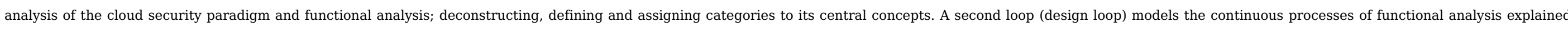

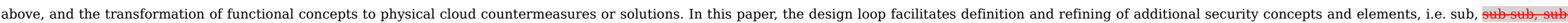

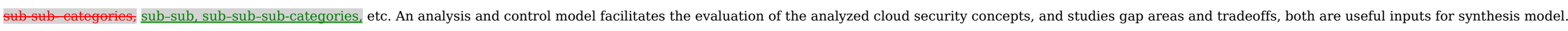

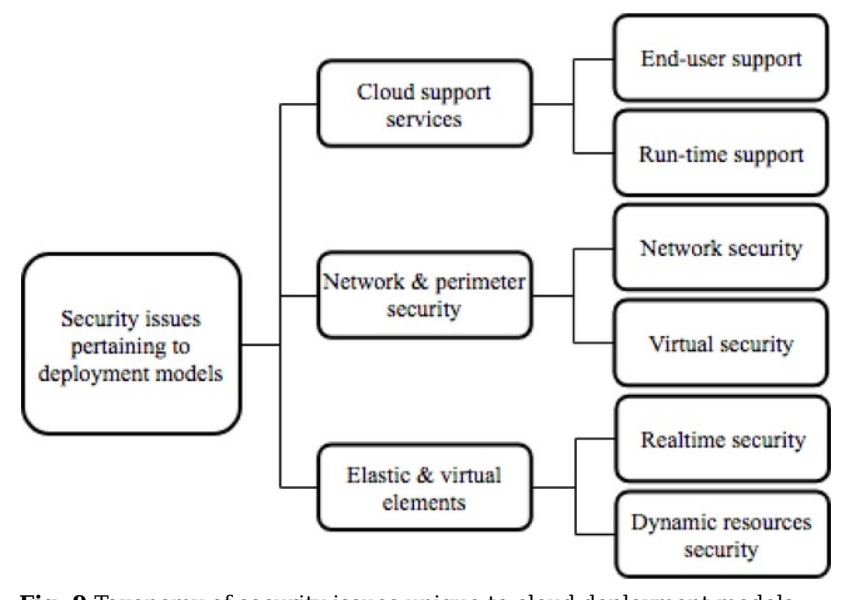

Fig. 9 Taxonomy of security issues unique to cloud deployment models. 


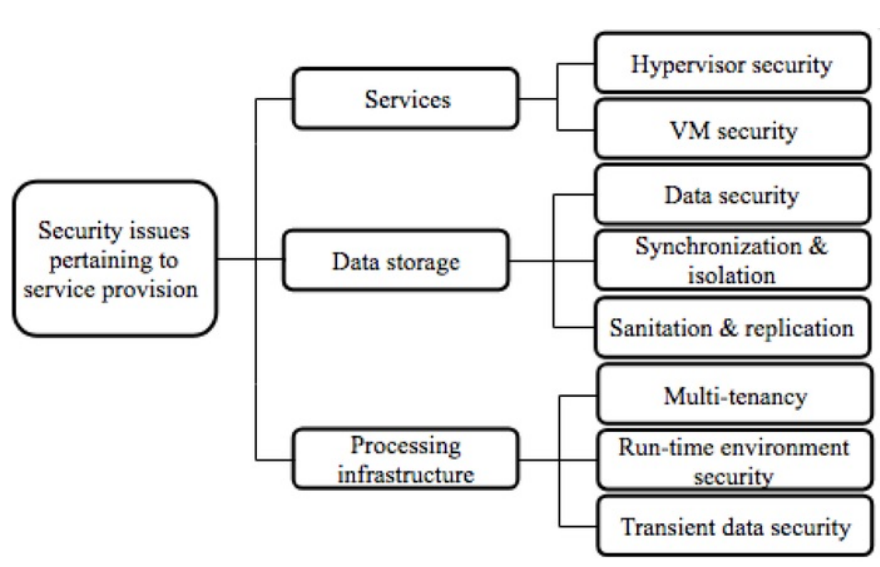

Fig. 10 Taxonomy of security issues unique to cloud service provision.

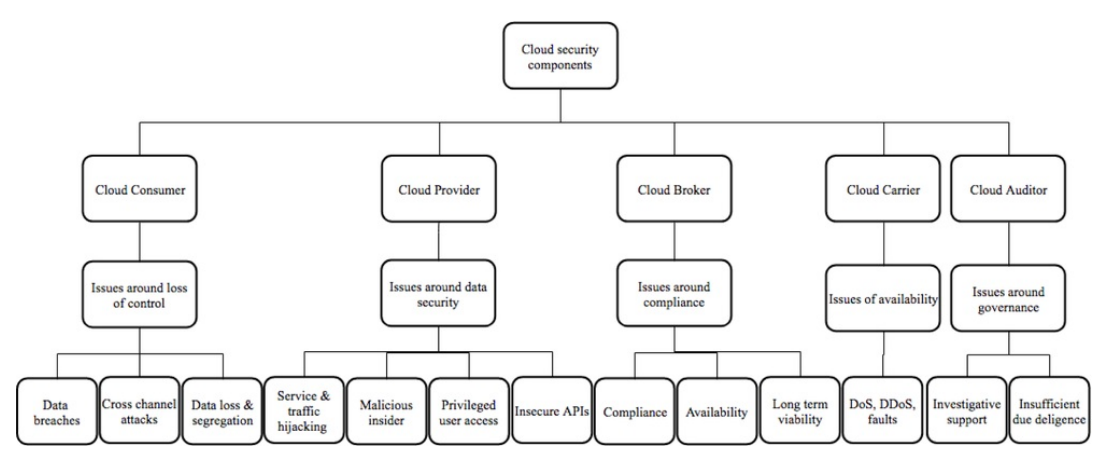

Fig. 11 Taxonomy of security challenges unique to cloud players.

\subsection{Taxonomy development}

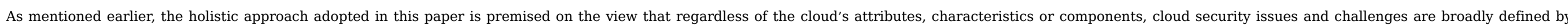

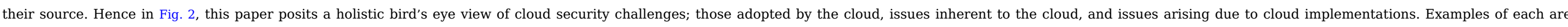
discussed in turn below.

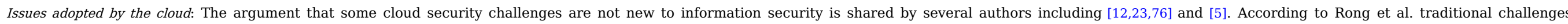

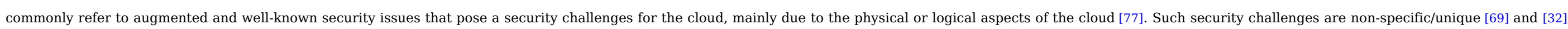

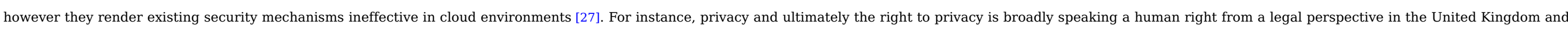

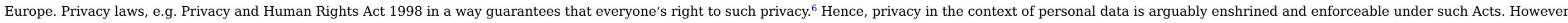

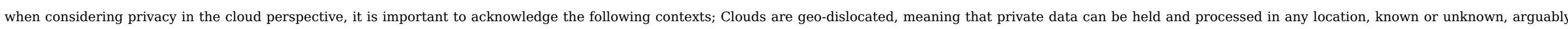

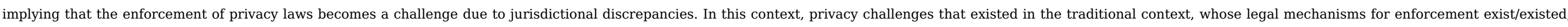
are leveraged in this new environment (see Fig. 3).

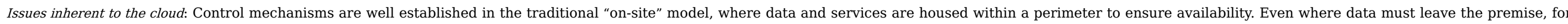

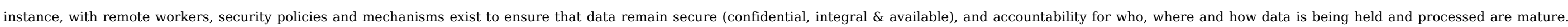

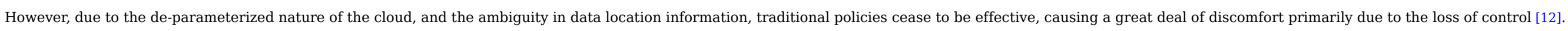




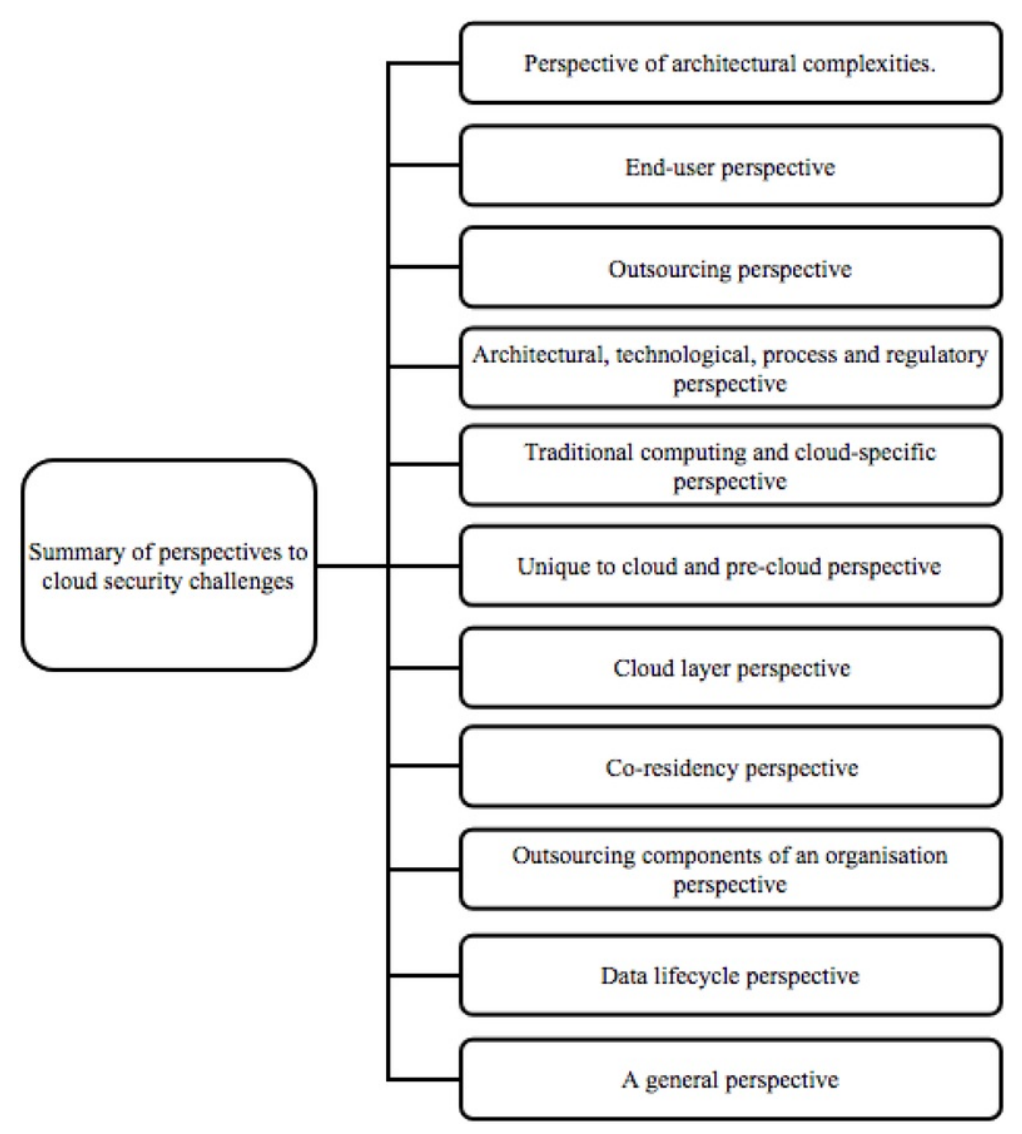

Fig. 12 A taxonomy of example multiple perspectives to cloud security issues. 


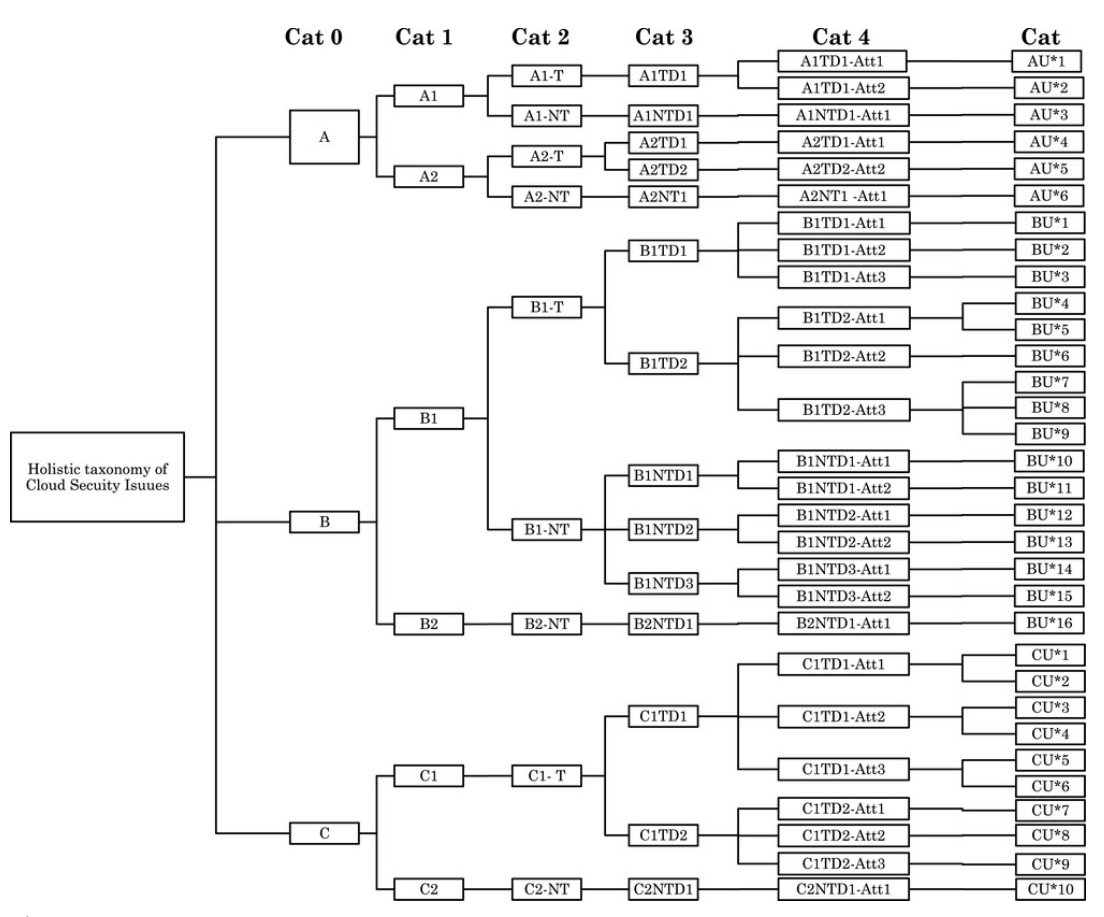

Fig. 13 Our proposed taxonomy for cloud security challenges.

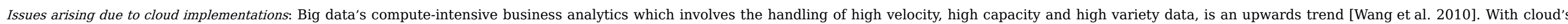

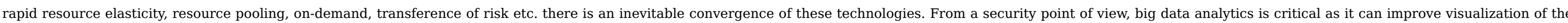

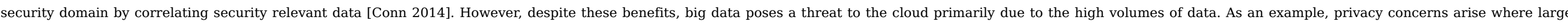

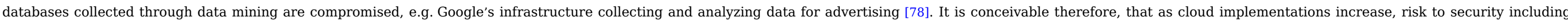
data breaches also increase. Furthermore, it is also conceivable that data security management policies as they exist in the traditional sense, will also be rendered ineffective in the cloud.

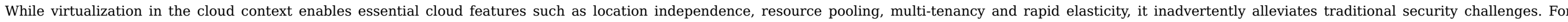

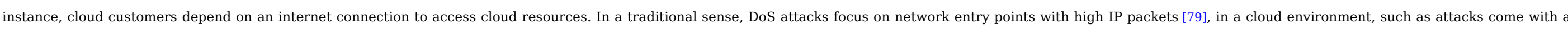

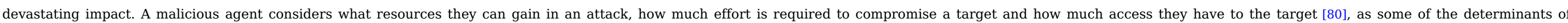

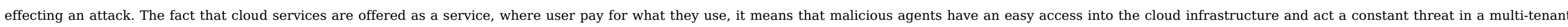
environment (see Figs. 4 and 5).

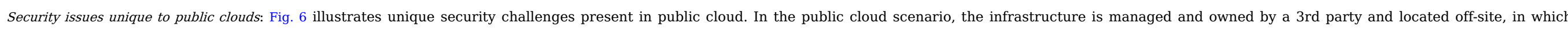

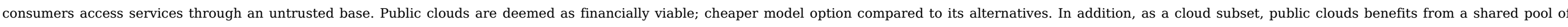

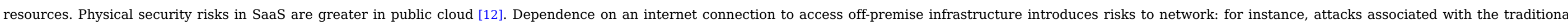

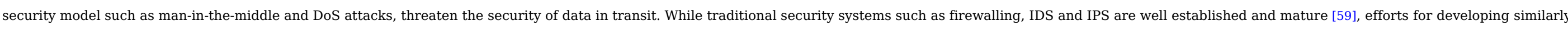

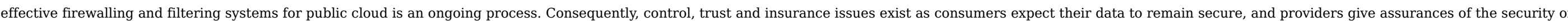
their services. 


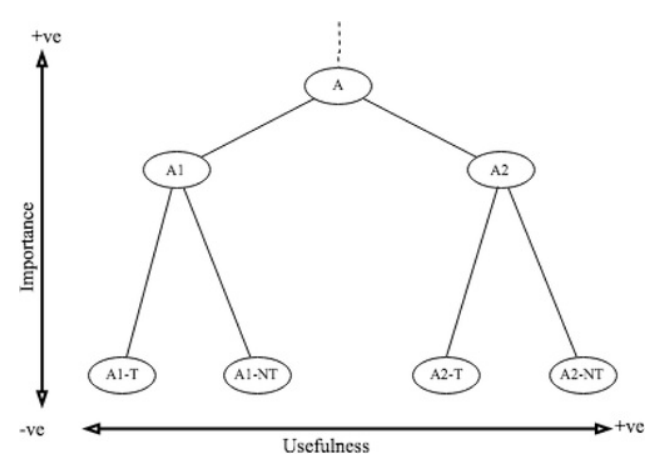

Fig. 14 Usefulness of levels and categories, sub-categories, sub-sub-categories, etc. increases (+vely and -vely) according to a use case scenario.

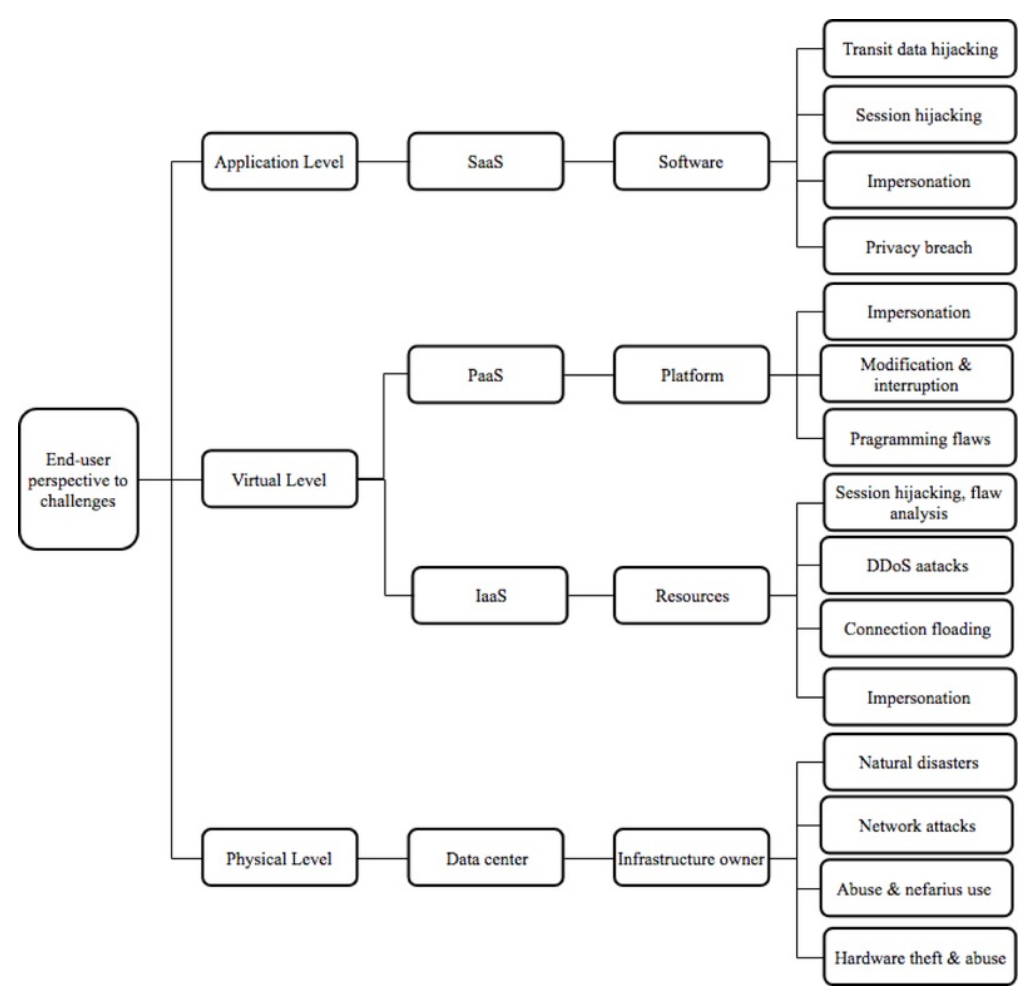

Fig. 15 An end user perspective taxonomy for cloud computing security challenges.

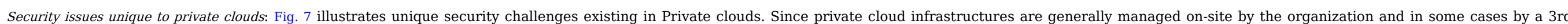

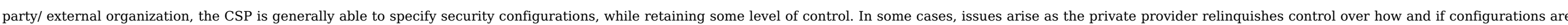

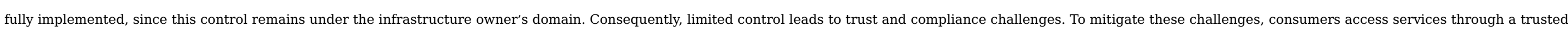
base. The offerings of public cloud versions over a private cloud, while presenting some flexibility and cost benefits, introduces security challenges associated with the public cloud. 


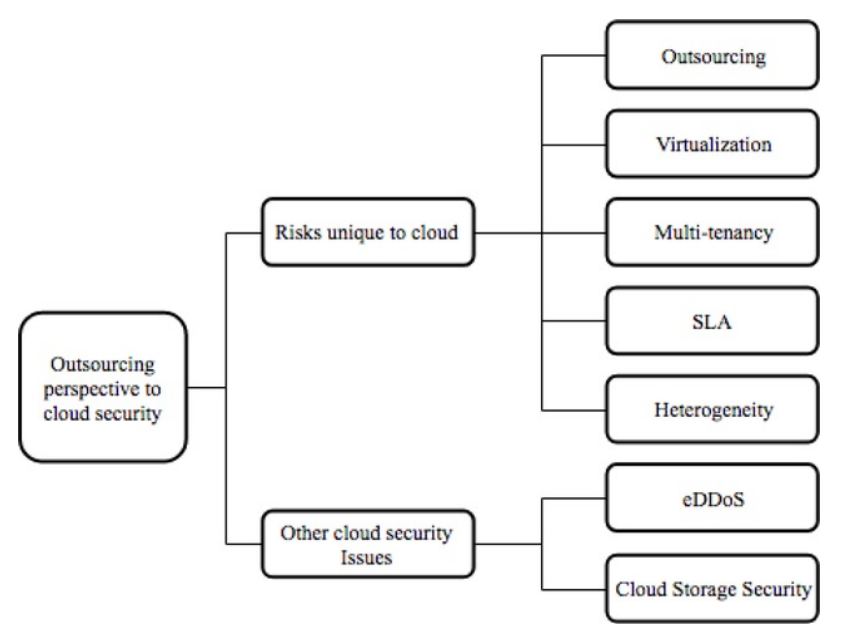

Fig. 16 An outsourcing perspective to cloud security challenges.

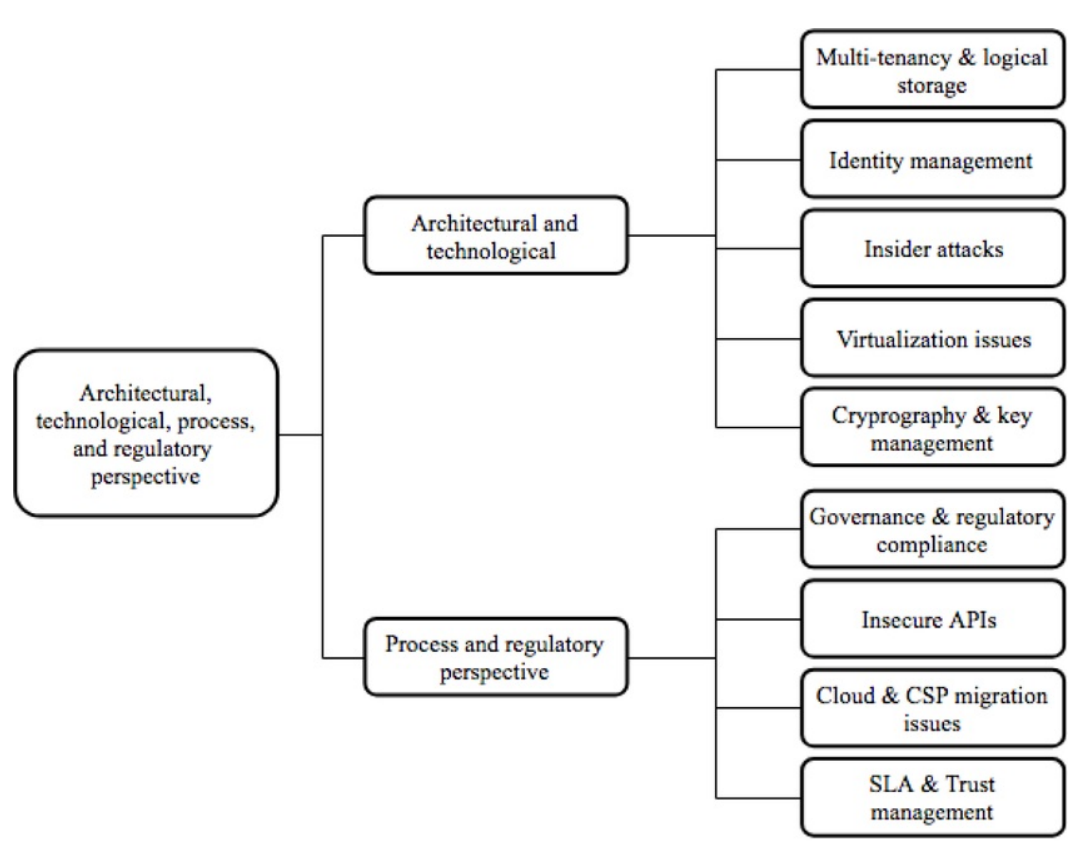

Fig. 17 Cloud security challenges from an architectural, technological, process and regulatory perspective. 


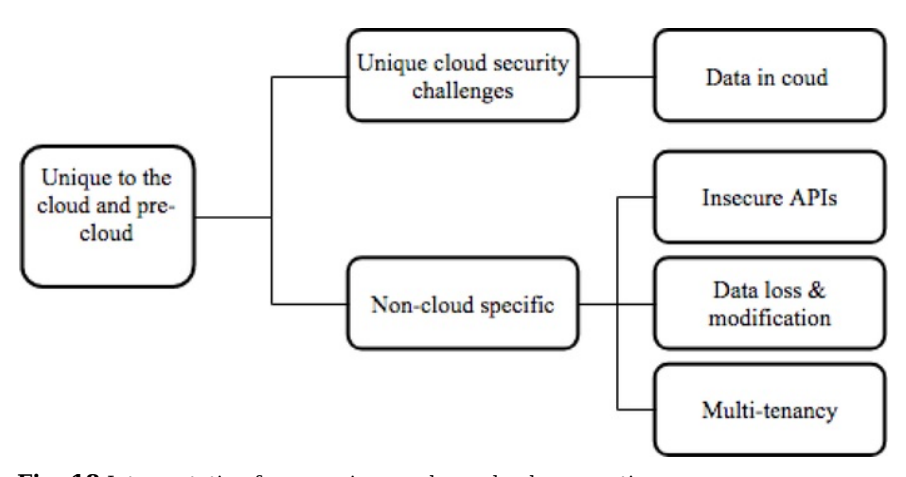

Fig. 18 Interpretation from a unique and pre-cloud perspective.

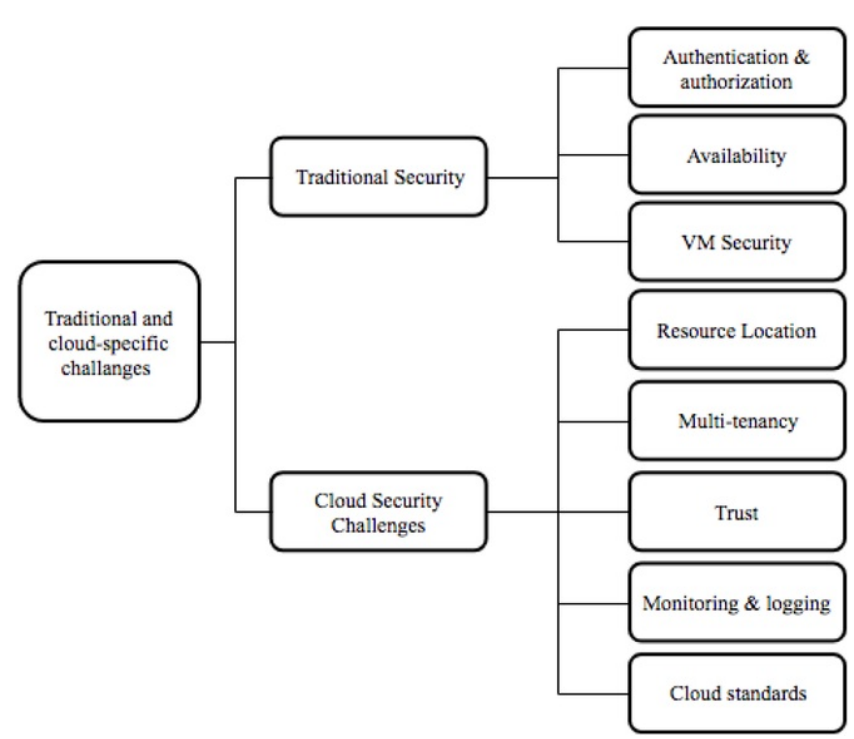

Fig. 19 A taxonomy illustrating our interpretation of traditional and cloud specific security challenges. 


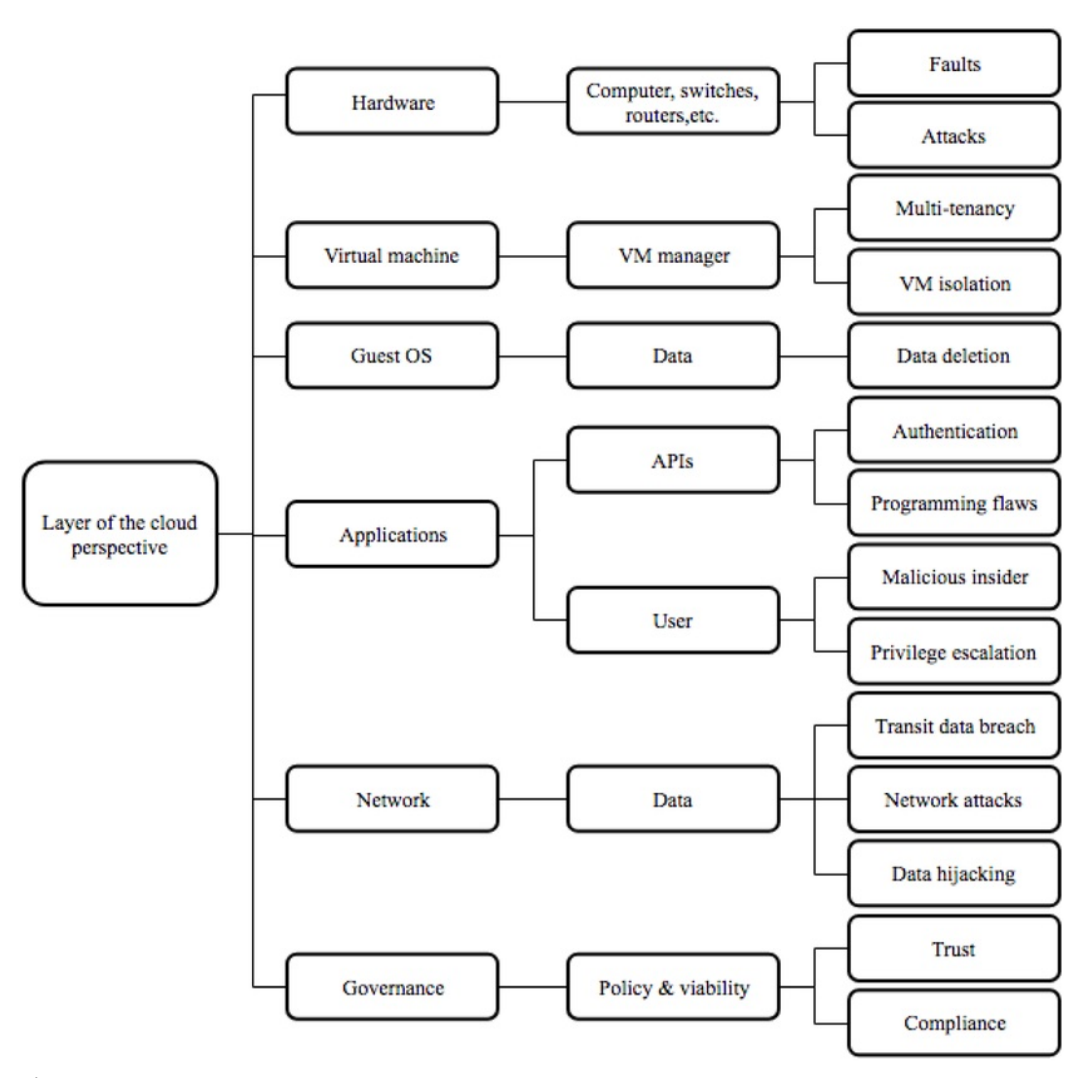

Fig. 20 A taxonomy illustrating our interpretation of cloud security challenges according to the layer of the cloud they affect.

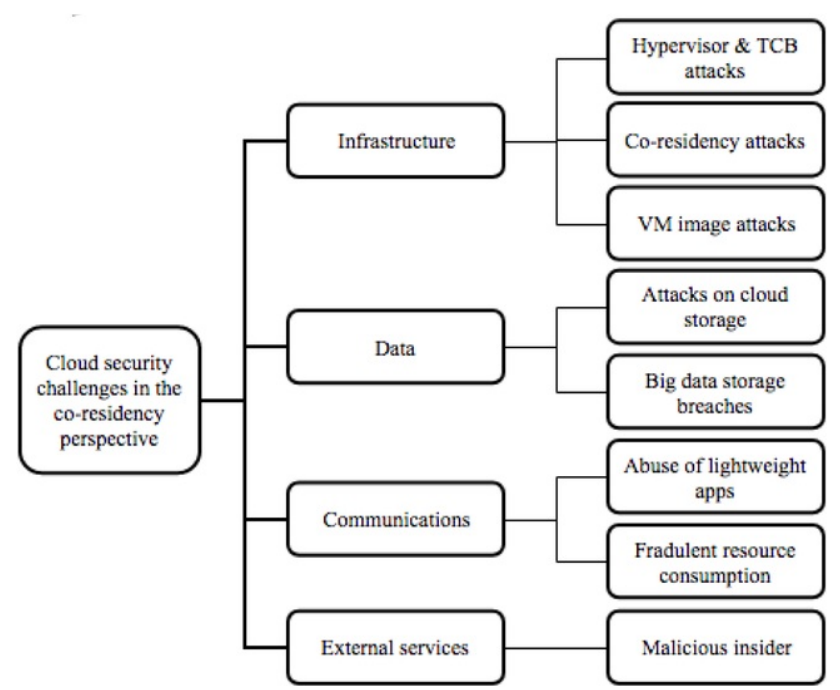

Fig. 21 A taxonomy of cloud security challenges from a co-residency perspective. 


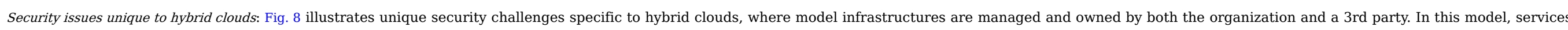

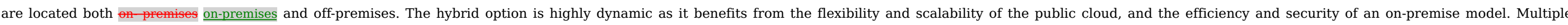

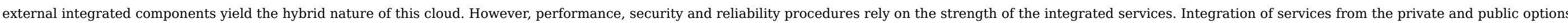

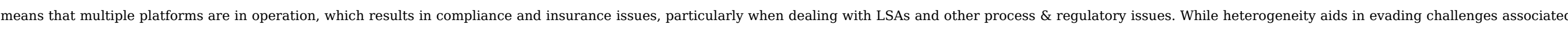
with platform lock-in, the same concept introduces integration challenges, i.e. security integration on a multi-vendor platform [81].

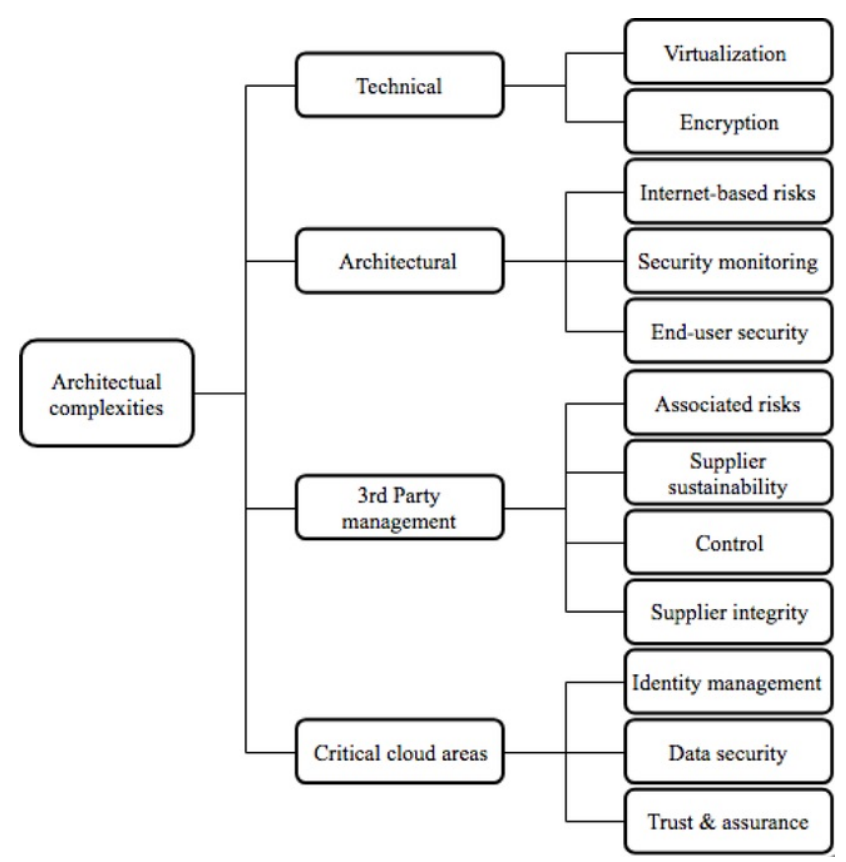

Fig. 22 A taxonomy illustrating cloud security challenges due to architectural complexities. 


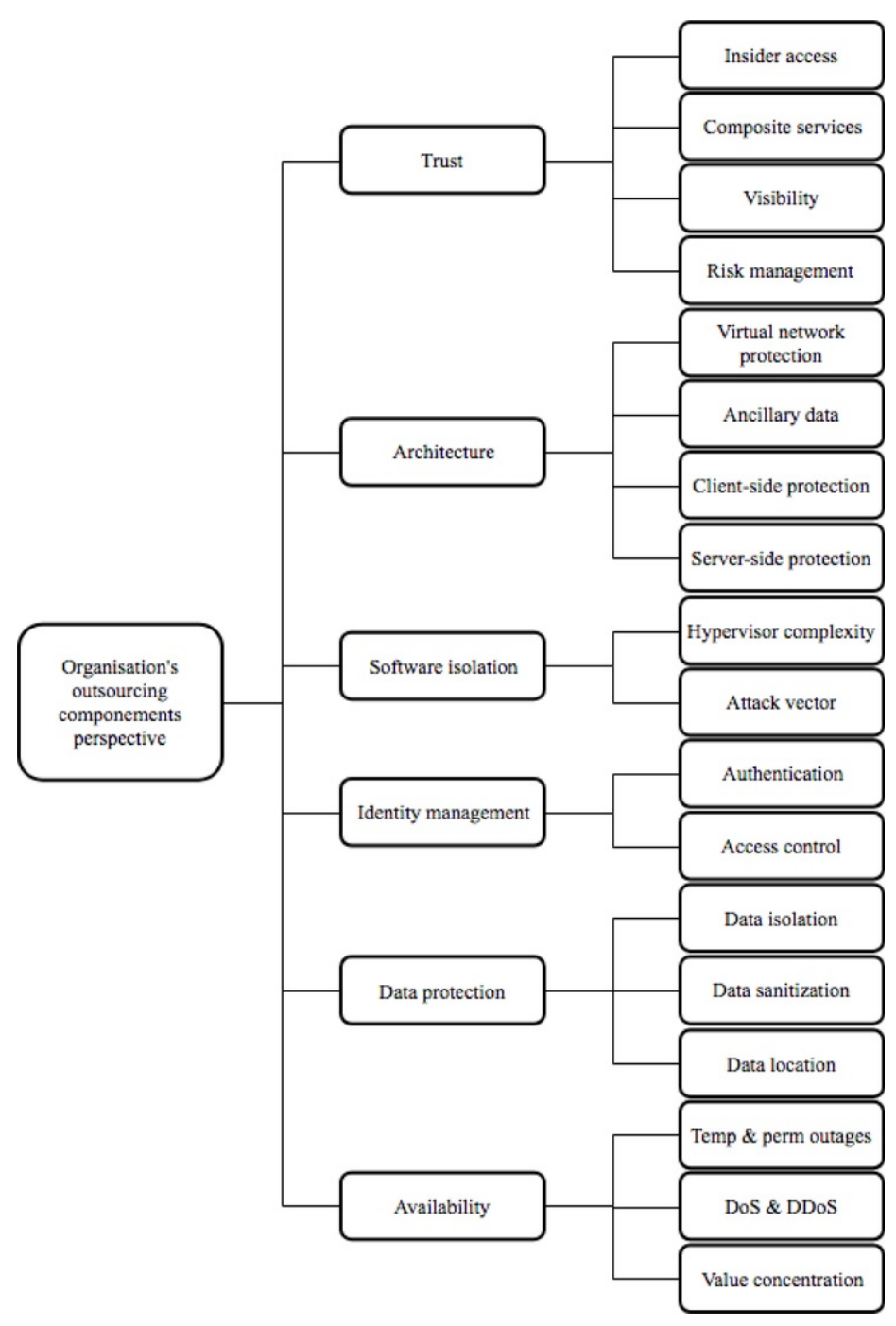

Fig. 23 A taxonomy of cloud security challenges highlighting the outsourcing components of an organization.

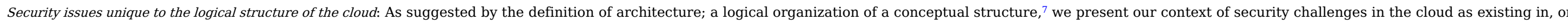

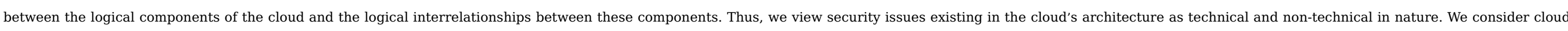

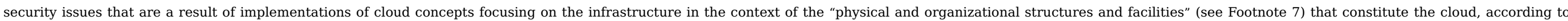

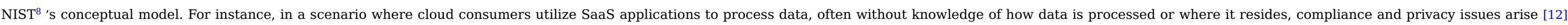

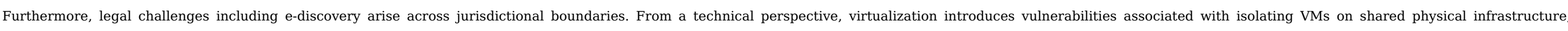

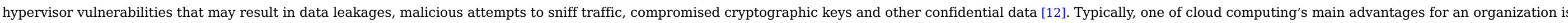

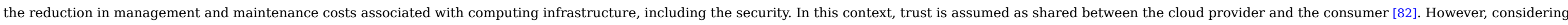

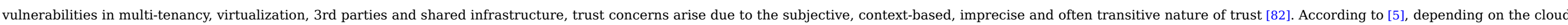




\subsection{Holistic taxonomy}

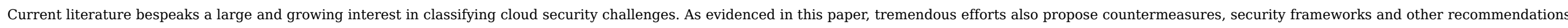

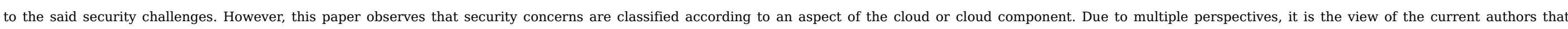
inadequately classified security issues will prevail despite much security spending. Fig. 12 summarizes these perspectives for reference's sake and place the current paper's proposition into context.

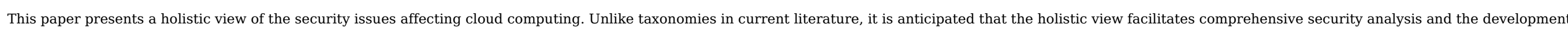

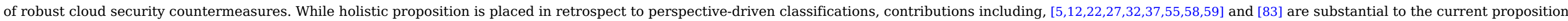

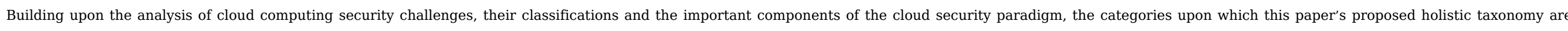

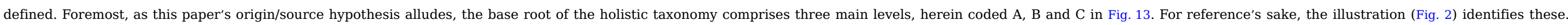

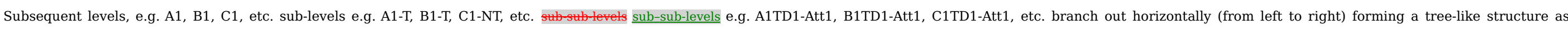

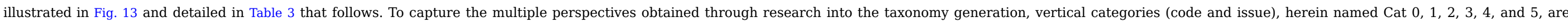

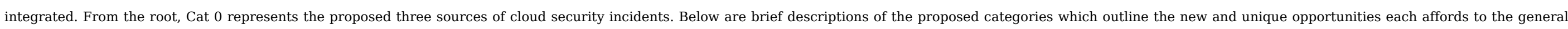
cloud-wide concept:

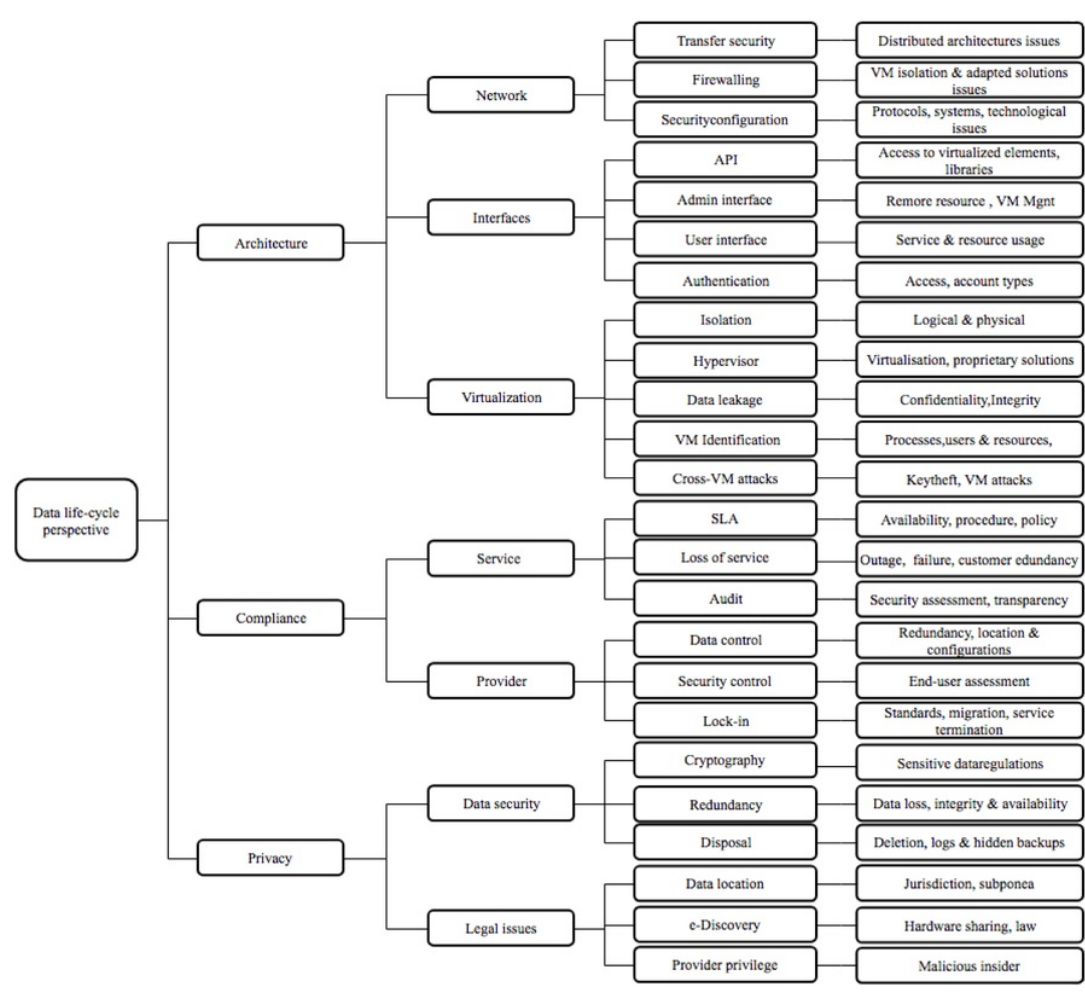

Fig. 24 A taxonomy illustrating cloud security challenges from a data lifecycle perspective 


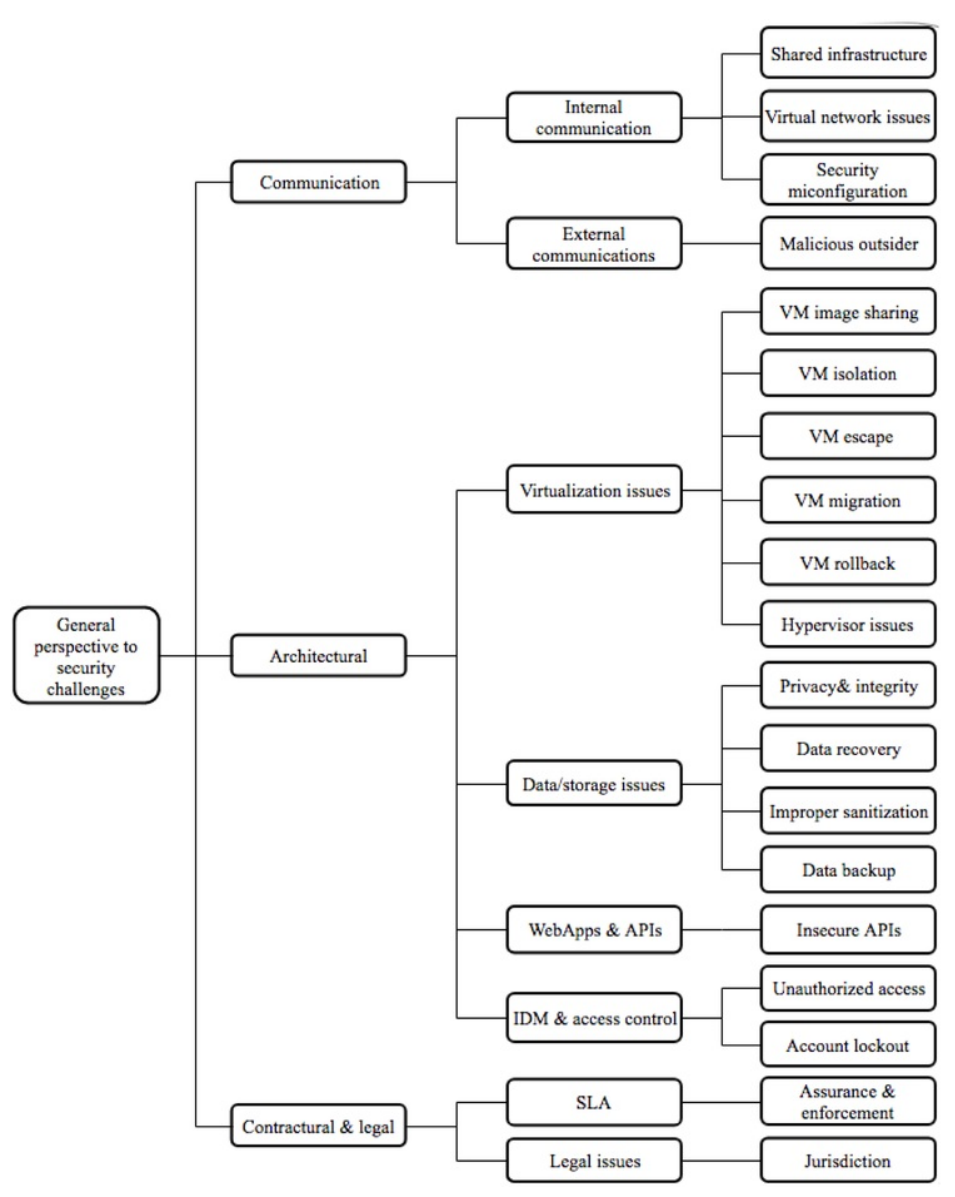

Fig. 25 A taxonomy of cloud security challenges by Ali et al. [2015].

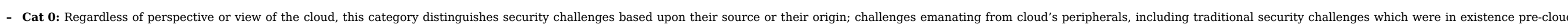

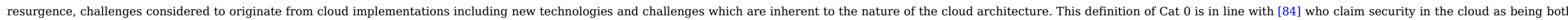

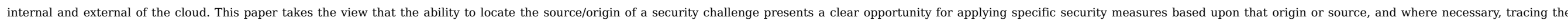
source. Hence Same Origin Policy (SOP) can be applied at Cat 0 , where monitoring of the original location of a request web request for instance, enabling validation of that requests [85].

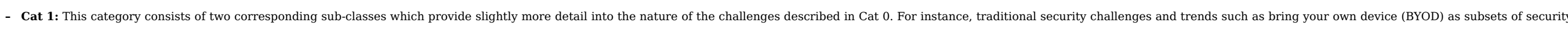

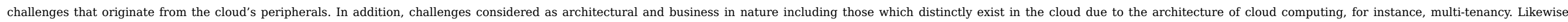
infrastructural and business challenges to describe issues that are a result of cloud implementations, for instance control over OS and application settings that is prevalent in SaaS.

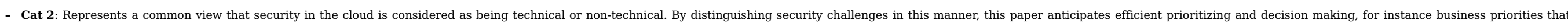
would attend to concerns such as governance and policy matters.

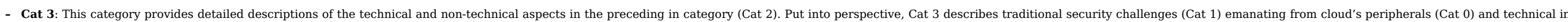

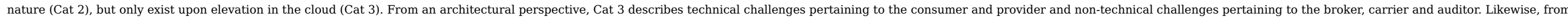
an infrastructure perspective, technical challenges pertain to deployment and service models. 


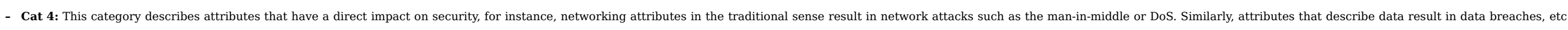
Virtual layer attributes result in multi-tenancy, virtual machine management (VMM) and hypervisor security issues.

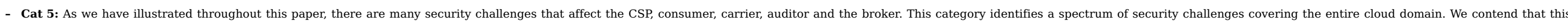
category is not exhaustive, thus, security challenges listed in this category exist for illustrative purposes.

Table 3 Detailed information for coding and issues in the categories and levels of the holistic taxonomy illustrated above in Fig. 13.

\begin{tabular}{|c|c|c|c|c|c|c|c|c|c|c|c|}
\hline \multicolumn{2}{|r|}{ CAT 0} & \multicolumn{2}{|r|}{ CAT 1} & \multicolumn{2}{|c|}{ CAT 2} & \multicolumn{2}{|r|}{ CAT 3} & \multicolumn{2}{|r|}{ CAT 4} & \multicolumn{2}{|r|}{ CAT 5} \\
\hline Code & Issue & Code & Issue & Code & Issue & Code & Issue & Code & Issue & Code & Unique Issues \\
\hline \multirow[t]{6}{*}{ A } & From cloud peripherals & A1 & Traditional & A1-T & Technical & A1TD1 & $\begin{array}{l}\text { Elevated traditional } \\
\text { issues }\end{array}$ & A1TD1-Att1 & Network & $\mathrm{AU}^{* 1}$ & Network attacks, DoS \\
\hline & & & & & & & & A1TD1-Att2 & Data & $\mathrm{AU}^{* 2}$ & Breaches \\
\hline & & & & A1-NT & Non-technical & A1NTD1 & $\begin{array}{l}\text { Processes \& } \\
\text { procedures }\end{array}$ & $\begin{array}{l}\text { A1NTD1- } \\
\text { Att1 }\end{array}$ & $\begin{array}{l}\text { Governance \& } \\
\text { Policy }\end{array}$ & $\mathrm{AU}^{* 3}$ & CIA \& AAA \\
\hline & & $\mathrm{A} 2$ & Trends & A2-T & Technical & A2TD1 & BYOD & A2TD1-Att1 & Data \& Network & $\mathrm{AU}^{*} 4$ & Malicious software \& outsider \\
\hline & & & & & & A2TD2 & Big data & A2TD2-Att2 & Data & $\mathrm{AU} * 5$ & Privacy, isolation, breaches \\
\hline & & & & A2-NT & Non-technical & A2NT1 & Outsourcing & $\begin{array}{l}\text { A2NT1 - } \\
\text { Att1 }\end{array}$ & 3rd parties & $\mathrm{AU}^{*} 6$ & Trust, control \& compliance \\
\hline \multirow[t]{16}{*}{ B } & $\begin{array}{l}\text { Existing in cloud } \\
\text { architecture }\end{array}$ & B1 & Architectural & B1-T & Technical & B1TD1 & Consumer & B1TD1-Att1 & Virtual & BU*1 & Multi-tenancy \\
\hline & & & & & & & & B1TD1-Att2 & Network & $\mathrm{BU} * 2$ & Malicious insider \\
\hline & & & & & & & & B1TD1-Att3 & Data storage & $\mathrm{BU} * 3$ & Malicious insider \\
\hline & & & & & & B1TD2 & Provider & B1TD2-Att1 & Virtual & $\mathrm{BU}^{*} 4$ & Hypervisor \\
\hline & & & & & & & & & & BU*5 & VM management \\
\hline & & & & & & & & B1TD2-Att2 & Network & $\mathrm{BU} * 6$ & Availability \\
\hline & & & & & & & & B1TD2-Att3 & Data storage & $\mathrm{BU} * 6$ & Isolation \\
\hline & & & & & & & & & & BU*7 & Deletion \\
\hline & & & & & & & & & & $\mathrm{BU} * 8$ & Breaches \\
\hline & & & & B1-NT & Non-technical & B1NTD1 & Broker & $\begin{array}{l}\text { B1NTD1- } \\
\text { Att1 }\end{array}$ & Policy & $\mathrm{BU} * 9$ & Compliance \\
\hline & & & & & & & & $\begin{array}{l}\text { B1NTD1- } \\
\text { Att2 }\end{array}$ & Governance & $\mathrm{BU} * 10$ & Data governance \\
\hline & & & & & & B1NTD2 & Carrier & $\begin{array}{l}\text { B1NTD2- } \\
\text { Att1 }\end{array}$ & Policy & BU*11 & Service reliability $\&$ availability \\
\hline & & & & & & & & Att2 & Governance & $\mathrm{BU} * 12$ & Privacy \\
\hline & & & & & & B1NTD3 & Auditor & $\begin{array}{l}\text { B1NTD3- } \\
\text { Att1 }\end{array}$ & Policy & & Monitoring \& logging \\
\hline & & & & & & & & Att2 & Governance & BU*13 & Assurance \\
\hline & & B2 & Business & B2-NT & Non-technical & B2NTD1 & $\begin{array}{l}\text { Processes \& } \\
\text { procedures }\end{array}$ & $\begin{array}{l}\text { B2NTD1- } \\
\text { Att1 }\end{array}$ & $\begin{array}{l}\text { Governance \& } \\
\text { Policy }\end{array}$ & $\mathrm{BU} * 14$ & LSAs \& Standards \\
\hline
\end{tabular}




\begin{tabular}{|c|c|c|c|c|c|c|c|c|c|c|c|}
\hline $\mathrm{C}$ & $\begin{array}{l}\text { Because of cloud } \\
\text { infrastructure }\end{array}$ & C1 & Infrastructural & $\begin{array}{l}\mathrm{E} 1 \mathrm{~T} \\
\mathrm{I}\end{array}$ & Technical & C1TD1 & Deployment & C1TD1-Att1 & Private & CU*1 & Complex attacks \\
\hline & & & & & & & & & & $\mathrm{CU} * 2$ & Availability-central failure \\
\hline & & & & & & & & C1TD1-Att2 & Public & $\mathrm{CU} * 3$ & Trust \\
\hline & & & & & & & & & & $\mathrm{CU}^{*} 4$ & Control \\
\hline & & & & & & & & C1TD1-Att3 & Hybrid & $\mathrm{CU} * 5$ & Compliance \& control \\
\hline & & & & & & & & & & $\mathrm{CU}^{*} 6$ & Fault propagation \\
\hline & & & & & & C1TD2 & Services & C1TD2-Att1 & Software & $\mathrm{CU} * 7$ & Data breaches, hijacking \& deletion \\
\hline & & & & & & & & C1TD2-Att2 & Platform & CU*8 & $\begin{array}{l}\text { Unauthorised Unauthorized users \& } \\
\text { insecure APIs }\end{array}$ \\
\hline & & & & & & & & C1TD2-Att3 & Infrastructure & CU*9 & DDoS, traffic analysis \& abuse of cloud \\
\hline & & $\mathrm{C} 2$ & Business & C2-NT & Non-technical & C2NTD1 & $\begin{array}{l}\text { Processes \& } \\
\text { procedures }\end{array}$ & $\begin{array}{l}\text { C2NTD1- } \\
\text { Att1 }\end{array}$ & $\begin{array}{l}\text { Governance \& } \\
\text { Policy }\end{array}$ & CU*10 & LSA \& standards \\
\hline
\end{tabular}

\subsection{Analysis}

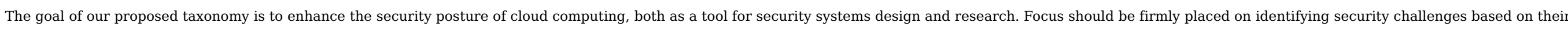

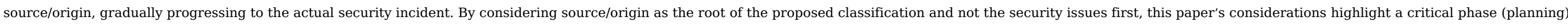

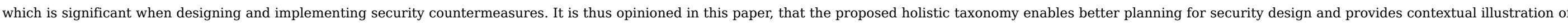

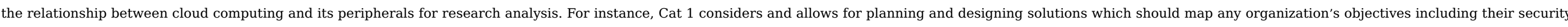

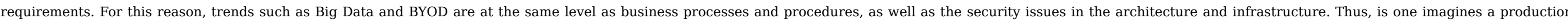

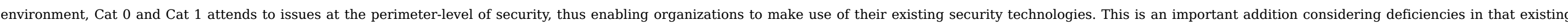

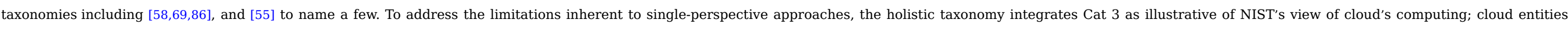
attributes and models.

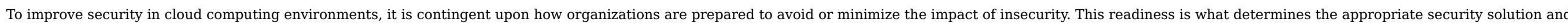

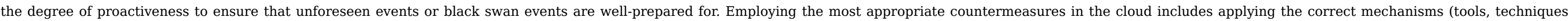

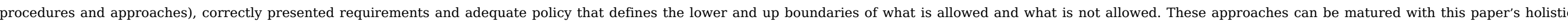

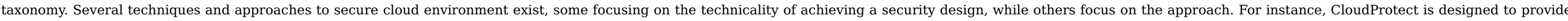

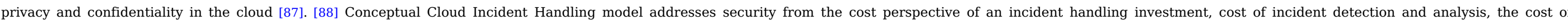
responding to an incident and post response cost.

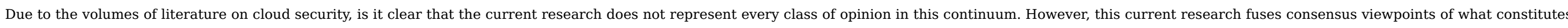

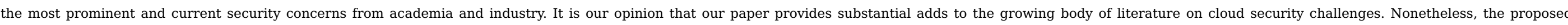

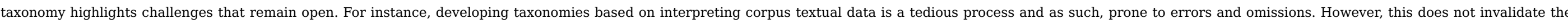

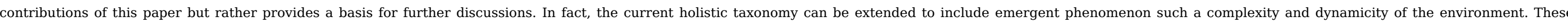

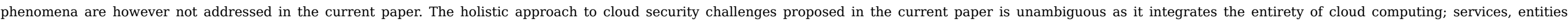

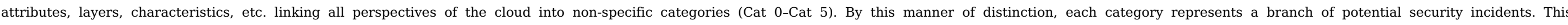
comprehensively organized taxonomy eliminates gaps introduced by perspective-driven views of the cloud and provides a basis for further research while aiding in developing security strategies.

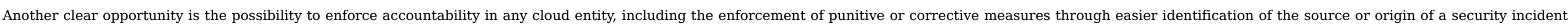




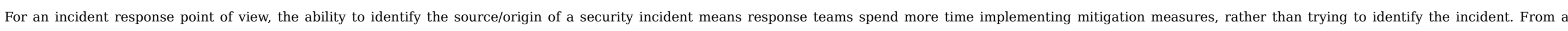
defensive security point-of-view, this proposed holistic taxonomy highlights security boundaries, including where security systems can be placed.

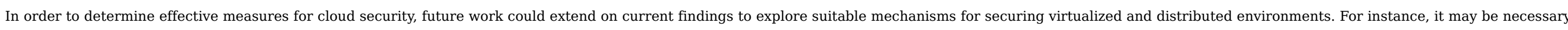

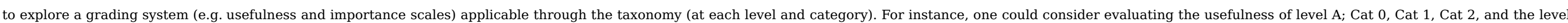

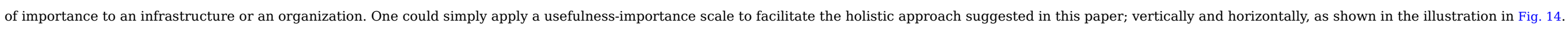

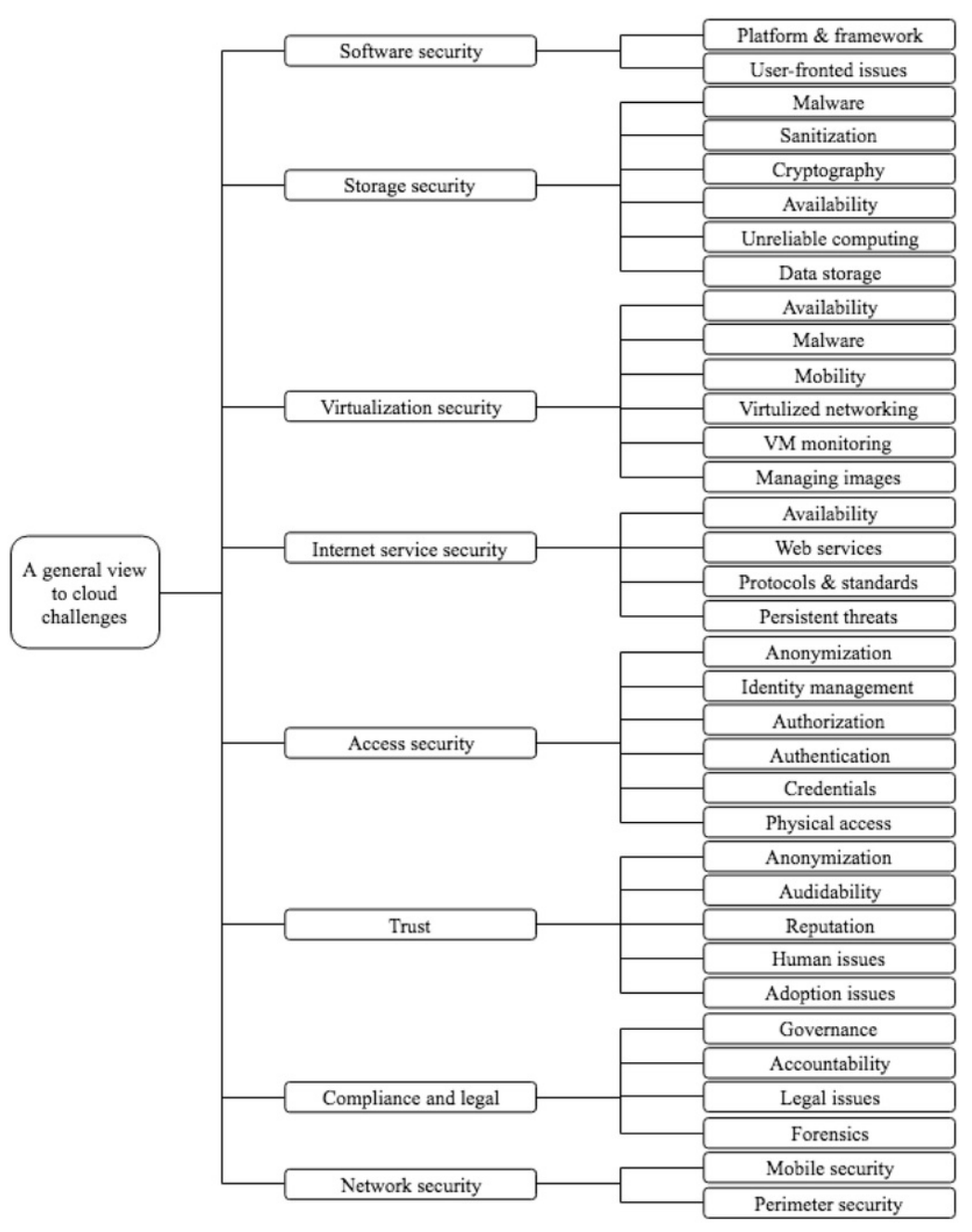

Fig. 26 A taxonomy illustrating our interpretation of a general view of cloud security challenges.

\section{Conclusions}

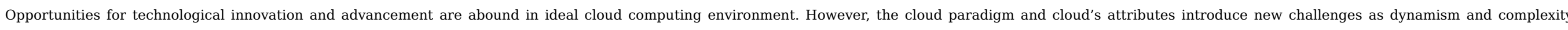

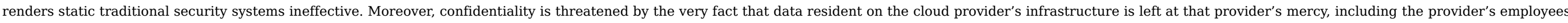

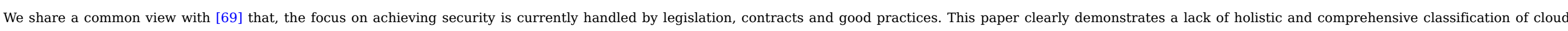




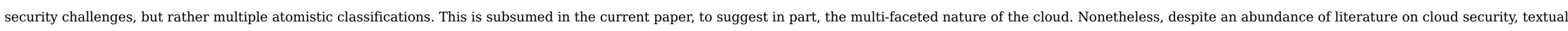

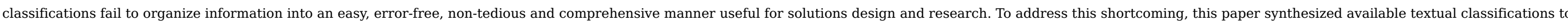

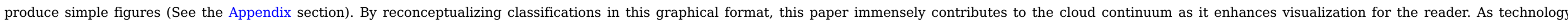

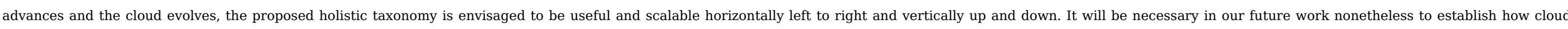

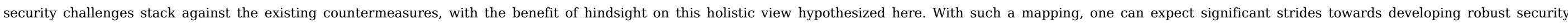
approaches that can scale enough to overhaul current cloud security issues.

\section{Declaration of Competing Interest}

The authors declare that they have no known competing financial interests or personal relationships that could have appeared to influence the work reported in this paper.

\section{Appendix}

See Figs. 15-26.

\section{References}

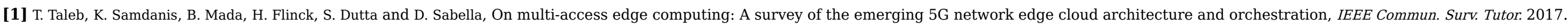

[2] Amazon Web Services, Amazon Elastic Compute Cloud, Amazon EC2, Vol. 2010, 2011, Amaz. Web Serv. LLC.

[3] A. Behl, K. Behl, An analysis of cloud computing security issues, in: Information and Communication Technologies (WICT), 2012 World Congress on, 2012, pp. 109-114.

[4] P. Mell and T. Grance, The NIST definition of cloud computing, 2011.

[5] D. Zissis and D. Lekkas, Addressing cloud computing security issues, Future Gener. Comput. Syst. 28 (3), 2012, 583-592.

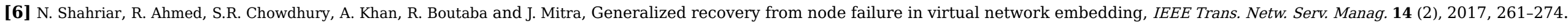

[7] Gartner, Gartner research, 2015, [Online]. Available: https://www.gartner.com/en. (Accessed 20 February 2015).

[8] Cloud Security Allience, Cloud security allience, 2015, [Online]. Available: https://cloudsecurityalliance.org/. (Accessed 20 February 2015).

[9] Verizon, 2015, [Online]. Available: https://enterprise.verizon.com/resources/reports/dbir/. (Accessed 20 February 2015).

[10] M. Johns, Code-injection vulnerabilities in web applications-exemplified at cross-site scripting, IT-Inform. Technol. Methoden Innov. Anwend. Inform. Informationstech. 53 (5), 2011, 256-260.

[11] F. Lombardi and R. Di Pietro, Secure virtualization for cloud computing, J. Netw. Comput. Appl. 34 (4), 2011, 1113-1122.

[12] S. Subashini and V. Kavitha, A survey on security issues in service delivery models of cloud computing, J. Netw. Comput. Appl. 34 (1), $2011,1-11$.

[13] A. Roy, S. Sarkar, R. Ganesan and G. Goel, Secure the cloud: From the perspective of a service-oriented organization, ACM Comput. Surv. 47 (3), 2015, 41:1-41:30.

[14] J.D. Howard and T.A. Longstaff, A Common Language for Computer Security Incidents, 1998, Sandia Natl. Lab.

[15] K. Malterud, Qualitative research: standards, challenges, and guidelines, Lancet 358 (9280), 2001, 483-488.

[16] S. Keele, Guidelines for performing systematic literature reviews in software engineering, 2007.

[17] C.A. Ardagna, R. Asal, E. Damiani and Q.H. Vu, From security to assurance in the cloud, ACM Comput. Surv. 48 (1), 2015, 1-50.

[18] C. Zott, R. Amit, L. Massa and Y. Zhu, Financial consumer protection and the global financial crisis, J. Consum. Res. 15 (2), 2012, 1-6. 
[19] F. Liu, et al., NIST Cloud Computing Reference Architecture, Vol. 500, 2011, NIST Spec. Publ., 292.

[20] T. Baars and M. Spruit, Analysing the security risks of cloud adoption using the SeCA model: A case study, J. UCS 18 (12), 2012, $1662-1678$.

[21] T. Ristenpart, E. Tromer, H. Shacham, S. Savage, Hey, you, get off of my cloud, in: Proc. 16th ACM Conf. Comput. Commun. Secur., CCS '09, 2009 , p. 199.

[22] Y. Liu, Y. Sun, J. Ryoo, S. Rizvi and A.V. Vasilakos, A survey of security and privacy challenges in cloud computing: Solutions and future directions, J. Comput. Sci. Eng. 2015.

[23] Y. Chen, V. Paxson and R.H. Katz, What's New About Cloud Computing Security, Rep. No.UCB/EECS-2010-5 January, vol. 20, no. 2010, 2010 , Univ. California; Berkeley, 2010-2015.

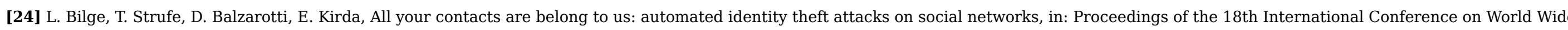
Web, 2009, pp. 551-560.

[25] F. Ahamed, S. Shahrestani and A. Ginige, Cloud computing: Security and reliability issues, Commun. IBIMA 2013, $2013,1$.

[26] M. Henning, API design matters, Queue 5 (4), 2007, 24-36.

[27] K. Hashizume, N. Yoshioka and E.B. Fernandez, Three misuse patterns for cloud computing, Secur. Eng. Cloud Comput. Approaches Tools 2013, 36-53.

[28] A. Aviram and B. Ford, 'No Title', Determ. timing channels Stat. Mult. clouds, 2010

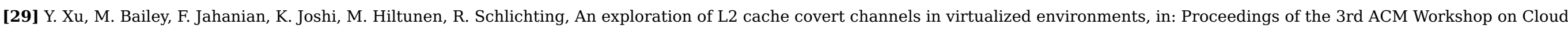
Computing Security Workshop, 2011, pp. 29-40.

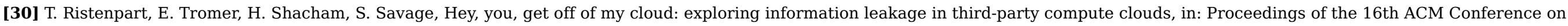
Computer and Communications Security, 2009, pp. 199-212.

[31] R. Krebs, C. Momm and S. Kounev, Architectural concerns in multi-tenant SaaS applications, Closer 12, 2012, 426-431.

[32] F. Shahzad, State-of-the-art survey on cloud computing security challenges, approaches and solutions, Proced. Comput. Sci. 37, 2014, 357-362.

[33] P.G. Dorey and A. Leite, Commentary: Cloud computing-A security problem or solution?, Inf. Secur. Tech. Rep. 16 (3), 2011, 89-96.

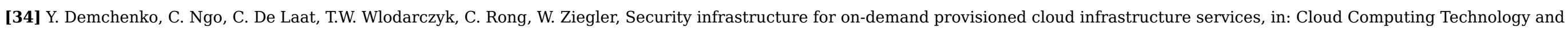
Science (CloudCom), 2011 IEEE Third International Conference on, 2011, pp. 255-263.

[35] A. Singh and K. Chatterjee, Cloud security issues and challenges: A survey, J. Netw. Comput. Appl. 79 (2016), 2017, 88-115.

[36] N.H. Hussein and A. Khalid, A survey of Cloud Computing Security challenges and solutions, Int. J. Comput. Sci. Inf. Secur. 14 (1), $2016,52$.

[37] M. Almorsy, J. Grundy and I. Müller, An analysis of the cloud computing security problem, 2016, arXiv:1609.01107 [cs].

[38] M. Daghmehchi Firoozjaei, J. (Paul) Jeong, H. Ko and H. Kim, Security challenges with network functions virtualization, Future Gener. Comput. Syst. 67, 2017, 315-324.

[39] R. Langner, Stuxnet: Dissecting a cyberwarfare weapon, Secur. Priv. IEEE 9 (3), 2011, 49-51.

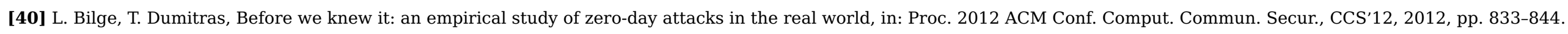

[41] B.B. Gupta and O.P. Badve, Taxonomy of DoS and DDoS attacks and desirable defense mechanism in a Cloud computing environment, Neural Comput. Appl. 2017.

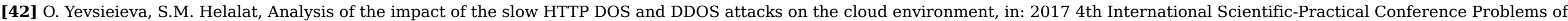
Infocommunications Science and Technology, PIC S and T 2017 - Proceedings, 2018. 


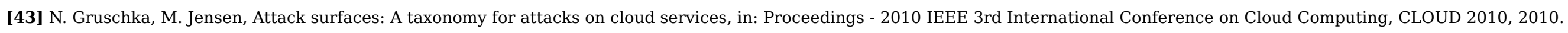

[44] A. Razzaq, K. Latif, H. Farooq Ahmad, A. Hur, Z. Anwar and P.C. Bloodsworth, Semantic security against web application attacks, Inf. SCi. (Ny) 2014.

[45] M. Ter Louw, V.N. Venkatakrishnan, Scripting attacks for existing browsers, in: Proceedings - IEEE Symposium on Security and Privacy, 2009.

[46] X. Li and Y. Xue, A survey on server-side approaches to securing web applications, ACM Comput. Surv. 2014.

[47] K.S. Schwaig, et al., Xml security, Secur. Priv. Trust Mod. Data Manag. 2 (2), 2014, 1-6.

[48] V.R. Mouli and K.P. Jevitha, Web services attacks and security- a systematic literature review, Procedia Comput. Sci. 2016.

[49] D. Hubbard and M. Sutton, Top Threats to Cloud Computing v1. 0, 2010, Cloud Secur. Alliance.

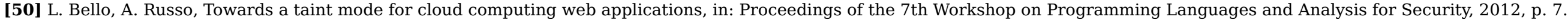

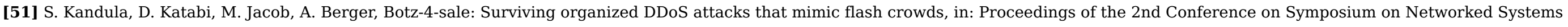
Design \& Implementation-Volume 2, 2005, pp. 287-300.

[52] A.C. Mora, Y. Chen, A. Fuchs, A. Lane, R. Lu and P. Manadhata, Top Ten Big Data Security and Privacy Challenges, Vol. 140, 2012, Cloud Secur. Alliance, November.

[53] S. Browne and M. Lang, Cloud security consciousness: A need for realisation, In: Entrepreneurial Small Firms, 2014.

[54] M.U. Farooq, M. Waseem, A. Khairi and S. Mazhar, A critical analysis on the security concerns of internet of things (IoT), Int. J. Comput. Appl. 111 (7), 2015.

[55] M. Ali, S.U. Khan and A.V. Vasilakos, Security in cloud computing: Opportunities and challenges, Inf. Sci. (Ny) 305, 2015, 357-383.

[56] M.T. Khorshed, A.B.M.S. Ali and S.A. Wasimi, Classifying different denial-of-service attacks in cloud computing using rule-based learning, Secur. Commun. Netw. 5 (11), 2012, 1235-1247.

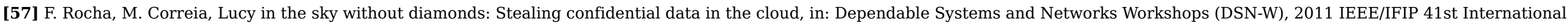
Conference on, 2011, pp. 129-134.

[58] A. Roy, S. Sarkar, R. Ganesan and G. Goel, Secure the cloud, ACM Comput. Surv. 47 (3), 2015, 1-30.

[59] N. Gonzalez, et al., A quantitative analysis of current security concerns and solutions for cloud computing, J. Cloud Comput. 1 (1), 2012, 1-18.

[60] M.T. Khorshed, et al., Data security and privacy protection issues in cloud computing, Future Gener. Comput. Syst. 2 (1), $2012,1-5$.

[61] W. Jansen and T. Grance, Guidelines on Security and Privacy in Public Cloud Computing, Vol. 800, 2011, NIST Spec. Publ, 144.

[62] A. Bisong and M. Rahman, An overview of the security concerns in enterprise cloud computing, 2011, arXiv Prepr. arXiv:1101.5613.

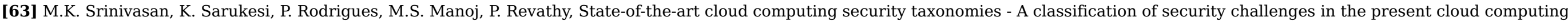
environment, in: Proc. Int. Conf. Adv. Comput. Commun. Informatics - ICACCI '12, 2012, p. 470.

[64] S. Ramgovind, M.M. Eloff and E. Smith, The management of security in cloud computing, Information Security for South Africa, ISSA, 2010, $2010,1-7$.

[65] S. Marston, Z. Li, S. Bandyopadhyay, J. Zhang and A. Ghalsasi, Cloud computing-The business perspective, Decis. Support Syst. 51 (1), 2011, 176-189.

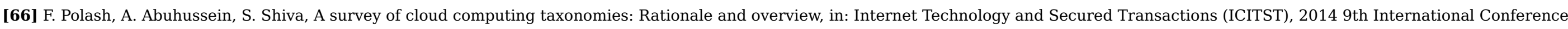
for, 2014, pp. 459-465.

[67] A. Botta, W. de Donato, V. Persico and A. Pescapé, Integration of cloud computing and internet of things: a survey, Future Gener. Comput. Syst. 56, $2016,684-700$. 
[68] M. Díaz, C. Martín and B. Rubio, State-of-the-art, challenges and open issues in the integration of Internet of things and cloud computing, J. Netw. Comput. Appl. 2016.

[69] M.D. Ryan, Cloud computing security: The scientific challenge, and a survey of solutions, J. Syst. Softw. 86 (9), 2013, 2263-2268.

[70] C. Rong, S.T. Nguyen and M.G. Jaatun, Beyond lightning: A survey on security challenges in cloud computing, Comput. Electr. Eng. 39 (1), 2013, 47-54.

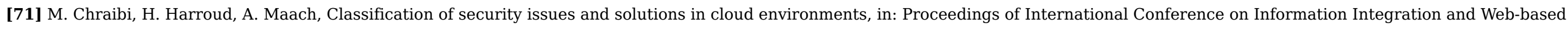
Applications \& Services, 2013, p. 560.

[72] W. Jansen, Cloud hooks: Security and privacy issues in cloud computing, in: System Sciences (HICSS), 2011 44th Hawaii International Conference on, 2011, pp. 1-10.

[73] I. Khalil, A. Khreishah and M. Azeem, Cloud computing security: A survey, Computers 3 (1), 2014, 1-35.

[74] N. Grant, T. Cadden, R. Mcivor and P. Humphreys, A taxonomy of manufacturing strategies in manufacturing companies in Ireland, J. Manuf. Technol. Manag. 2013.

[75] R.C. Nickerson, U. Varshney and J. Muntermann, A method for taxonomy development and its application in information systems, Eur. J. Inf. Syst. 2013.

[76] S. Pearson, A. Benameur, Privacy, security and trust issues arising from cloud computing, in: 2010 IEEE Second Int. Conf. Cloud Comput. Technol. Sci., 2010, pp. 693-702.

[77] R. Yu, et al., Cooperative resource management in cloud-enabled vehicular networks, IEEE Trans. Ind. Electron. 62 (12), $2015,7938-7951$.

[78] R. Chow, et al. Controlling data in the cloud, in: Proc. 2009 ACM Work. Cloud Comput. Secur., CCSW '09, 2009 , p. 85.

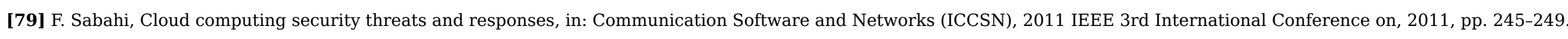

[80] B. Grobauer, T. Walloschek and E. Stocker, Understanding cloud computing vulnerabilities, Secur. Priv. IEEE 9 (2), $2011,50-57$.

[81] H. Takabi, J.B.D. Joshi and G.-J. Ahn, Security and privacy challenges in cloud computing environments, IEEE Secur. Priv. (6), 2010, $24-31$.

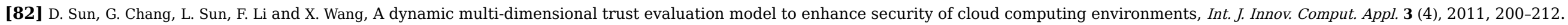

[83] D. Huang and H. Wu, Mobile cloud computing taxonomy, In: Mobile Cloud Computing, 2018.

[84] B.Y.M. Armbrust, et al., of cloud computing, Commun. ACM 53 (4), 2010, 50-59.

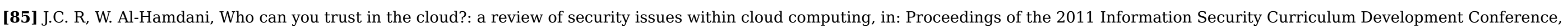
2011, pp. 15-19.

[86] D.A.B. Fernandes, L.F.B. Soares, J.V. Gomes, M.M. Freire and P.R.M. Inácio, Security issues in cloud environments: a survey, Int. J. Inf. Secur. 13 (2), 2014 , 113-170.

[87] C.A. Ardagna, R. Asal, E. Damiani and Q.H. Vu, From security to assurance in the cloud: A survey, ACM Comput. Surv. 48 (1), $2015,2$.

[88] N.H.A. Rahman and K.-K.R. Choo, A survey of information security incident handling in the cloud, Comput. Secur. 49, 2015, 45-69.

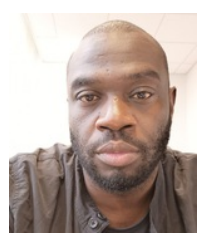

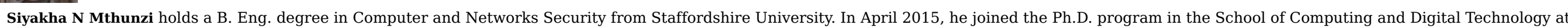

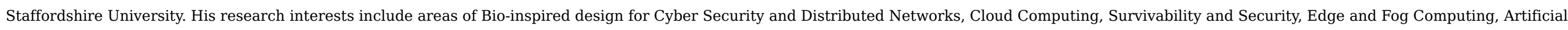
Intelligence, and Internet of things. He is a Fellow of the Higher Education Academy. 


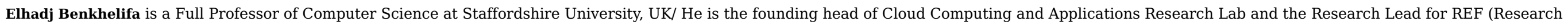

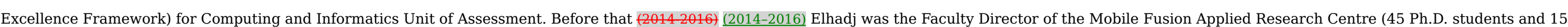

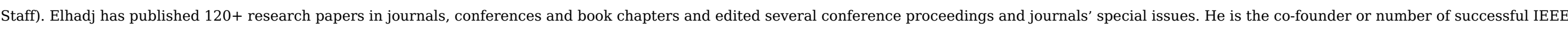

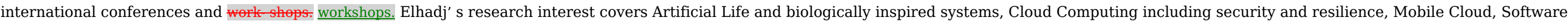

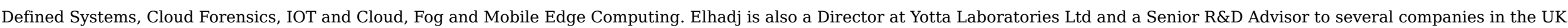

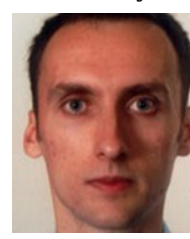

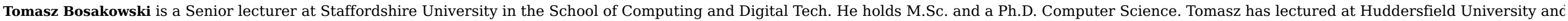

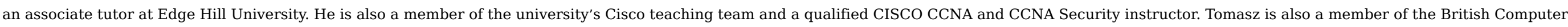
Society (BCS), and Associate Member of the BCS (AMBCS), 2011.

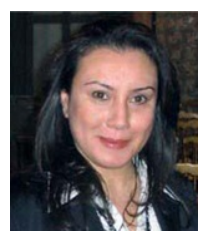

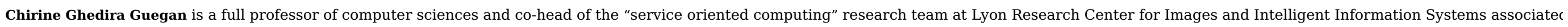

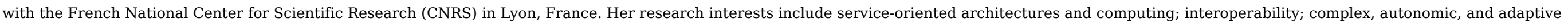
systems; context-aware computing; data services; privacy; and cloud computing. Chirine Ghedira Guegan has a research habilitation in computer science from Université de Lyon 1.

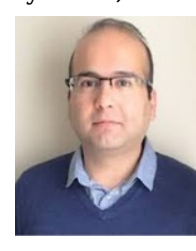

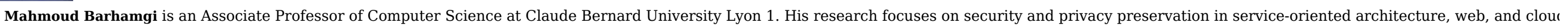
environments. Barhamgi received a Ph.D. in information and communication technology from Claude Bernard University Lyon 1. Contact him at mahmoud.barhamgi@univ-lyon1.fr.

\section{Footnotes}

$\mathbf{1}_{\text {https://cloudsecurityalliance.org/download/the-notorious-nine-cloud-computing-top-threats-in-2013/. }}$

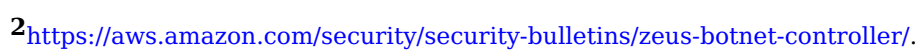

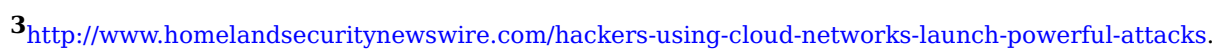

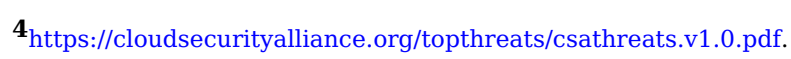


$\mathbf{5}_{\text {http://www.gartner.com/technology/home.jsp. }}$

$\mathbf{6}_{\text {https://www.equalityhumanrights.com/en/human-rights-act/article-8-respect-your-private-and-family-life. }}$

$7_{\text {https://en.oxforddictionaries.com/definition/architecture. }}$

$\mathbf{8}_{\text {http://www.nist.gov/itt/idms/. }}$

\section{Queries and Answers}

Query: Please check whether the designated corresponding author is correct, and amend if necessary.

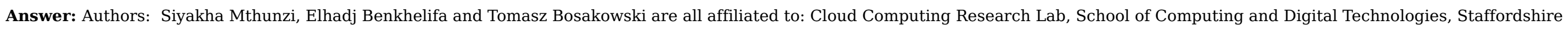
University, Stoke-on-Trent, Staffordshire, ST4 2DE, UK (one affiliation) .

Query: Please check the corresponding address, and correct if necessary.

Answer: The corresponding author is: Elhadj Benkhelifa, School of Computing and Digital Technologies, College Road, Stoke on Trent, ST4 2DE, UK. e.benkhelifa@staffs.ac.uk

Query: Please check the e-mail address(es) of the corresponding author(s).

Answer: e.benkhelifa@staffs.ac.uk

Query: Highlights should only consist of 85 characters per bullet point, including spaces. The highlights provided are too long; please edit them to meet the requirement.

Answer: not sure on this

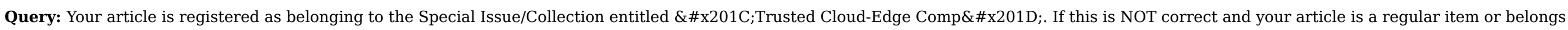
to a different Special Issue please contact immediately prior to returning your corrections.

Answer: correct

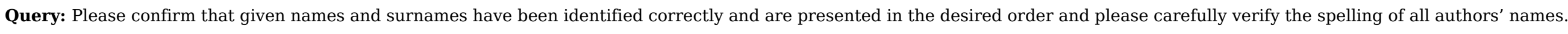

Answer: Yes

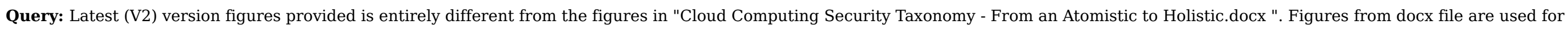
processing. Please check whether the figures are ok.

Answer: fine

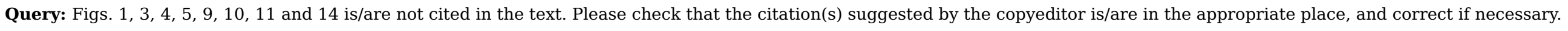
Answer: yes fine

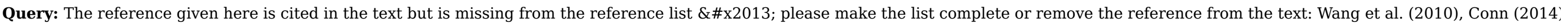
Ali et al. (2015).

Answer: please remove. they are left in the paper by mistake

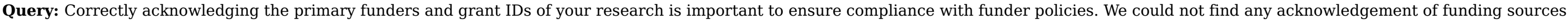
in your text. Is this correct?

Answer: correct

Query: Please confirm the title for Ref. [28]

Answer: this is the full and correct reference 
